\title{
Modulation of Jupiter's plasma flow, polar currents, and auroral precipitation by solar wind-induced compressions and expansions of the magnetosphere: a simple theoretical model
}

\author{
S. W. H. Cowley ${ }^{1}$, J. D. Nichols ${ }^{2}$, and D. J. Andrews ${ }^{1}$ \\ ${ }^{1}$ Department of Physics \& Astronomy, University of Leicester, Leicester LE1 7RH, UK \\ ${ }^{2}$ Center for Space Physics, Boston University, Boston, MA 02215, USA
}

Received: 10 February 2007 - Accepted: 24 May 2007 - Published: 29 June 2007

\begin{abstract}
We construct a simple model of the plasma flow, magnetosphere-ionosphere coupling currents, and auroral precipitation in Jupiter's magnetosphere, and examine how they respond to compressions and expansions of the system induced by changes in solar wind dynamic pressure. The main simplifying assumption is axi-symmetry, the system being modelled principally to reflect dayside conditions. The model thus describes three magnetospheric regions, namely the middle and outer magnetosphere on closed magnetic field lines bounded by the magnetopause, together with a region of open field lines mapping to the tail. The calculations assume that the system is initially in a state of steady diffusive outflow of iogenic plasma with a particular equatorial magnetopause radius, and that the magnetopause then moves rapidly in or out due to a change in the solar wind dynamic pressure. If the change is sufficiently rapid ( $\sim 2-3 \mathrm{~h}$ or less) the plasma angular momentum is conserved during the excursion, allowing the modified plasma angular velocity to be calculated from the radial displacement of the field lines, together with the modified magnetosphere-ionosphere coupling currents and auroral precipitation. The properties of these transient states are compared with those of the steady states to which they revert over intervals of $\sim 1-2$ days. Results are shown for rapid compressions of the system from an initially expanded state typical of a solar wind rarefaction region, illustrating the reduction in total precipitating electron power that occurs for modest compressions, followed by partial recovery in the emergent steady state. For major compressions, however, typical of the onset of a solar wind compression region, a brightened transient state occurs in which super-rotation is induced on closed field lines, resulting in a reversal in sense of the usual magnetosphereionosphere coupling current system. Current system reversal results in accelerated auroral electron precipitation occurring
\end{abstract}

Correspondence to: S. W. H. Cowley

(swhc1@ion.le.ac.uk) in the outer magnetosphere region rather than in the middle magnetosphere as is usual, with peak energy fluxes occurring just poleward of the boundary between the outer and middle magnetosphere. Plasma sub-corotation is then re-established as steady-state conditions re-emerge, together with the usual sense of flow of the closed field current system and renewed but weakened accelerated electron precipitation in the middle magnetosphere. Results for rapid expansions of the system from an initially compressed state typical of a solar wind compression region are also shown, illustrating the enhancement in precipitating electron power that occurs in the transient state, followed by partial reduction as steady conditions re-emerge.

Keywords. Magnetospheric physics (Auroral phenomena; Magnetosphere-ionosphere interactions; Planetary magnetospheres; Solar wind-magnetosphere interactions)

\section{Introduction}

Considerable progress has been made in recent years in understanding the properties and origins of Jupiter's polar auroral emissions. Studies of the structure and morphology of the auroras have focussed on ultraviolet (UV) images obtained since 1992 by the Hubble Space Telescope (HST) (see e.g. the review by Clarke et al., 2004), most recently on high-resolution images and spectra from the Space Telescope Imaging Spectrograph (STIS) (Clarke et al., 2002; Grodent et al., 2003a, b). These images have clearly delineated components related to moon footprints, the main auroral oval, and variable polar emissions at highest latitudes, of which we focus here on the latter two. The UV spectra of these emissions have shown that they are produced by precipitating electrons with mean energies typically in the range $\sim 50$ $150 \mathrm{keV}$ (Gustin et al., 2004). Ground-based measurements of Doppler-shifted infrared (IR) emissions from $\mathrm{H}_{3}^{+}$ions in

Published by Copernicus Publications on behalf of the European Geosciences Union. 
Jupiter's polar ionosphere have also been used to measure related significant departures of the ionospheric plasma flow from rigid corotation with the planet (Rego et al., 1999; Stallard et al., 2001, 2003).

Substantial theoretical progress has also been made in understanding these emissions and related plasma flows. Bunce and Cowley (2001), Hill (2001), and Southwood and Kivelson (2001) independently suggested that the main oval is related to the current system associated with the communication of angular momentum between the planet's polar atmosphere and the middle magnetosphere plasma disc of iogenic origin. The first quantitative model derived on this basis was presented by Cowley and Bunce (2001), who combined an empirical model of the plasma angular velocity profile in Jupiter's middle magnetosphere with a realistic magnetic field geometry to calculate the magnetosphere-ionosphere coupling currents flowing in the region. They showed that the field-aligned current was directed upward, out of the planet's ionosphere, throughout the middle magnetosphere, and of sufficient peak magnitude that downward acceleration of hot magnetospheric electrons by field-aligned voltages to energies of $\sim 100 \mathrm{keV}$ is required to carry it. The precipitating electron energy flux is thereby increased to values sufficient to produce a UV aurora of several hundred $\mathrm{kR}$ intensity in a latitudinal region $\sim 1^{\circ}$ wide at $\sim 15^{\circ}$ co-latitude from the magnetic pole, thus corresponding to the main oval emission. The upward current and auroral region maps magnetically to the middle magnetosphere beyond $\sim 20 R_{J}$ in the equatorial plane, where $R_{J}$ is Jupiter's equatorial radius $(71373 \mathrm{~km})$.

Subsequent development of middle magnetosphereionosphere coupling models has incorporated plasma angular velocity models calculated self-consistently from the outflow of iogenic plasma using the theory of Hill (1979) and Pontius (1997), explored the effect of a wide range of system parameters (plasma outflow rate and ionospheric Pedersen conductivity) on the solutions, calculated the feedback of electron precipitation on auroral conductivity, and considered the magnetosphere-ionosphere decoupling effects associated with the auroral field-aligned voltages (Cowley et al., 2002, 2003a; Nichols and Cowley, 2003, 2004, 2005). All of these models considered only the inner and middle magnetosphere, however, such that overall closure of the current system could not be investigated. Recently, Cowley et al. (2005) have constructed an axi-symmetric model of the whole jovian polar region, starting from a plasma angular velocity profile based on the above middle magnetosphere models, observational data, and the physical discussion of Cowley et al. (2003b). They showed that the expected pattern of field-aligned currents consists of four regions, with downward-directed current flowing in the strongly subcorotating region of open field lines mapping to the tail, reversing to a sheet of upward-directed current at the boundary between open and closed field lines where the angular velocity increases on closed field lines, followed by a resumption of downward-directed current in the outer magne- tosphere, and then upward-directed current once more in the middle magnetosphere where the angular velocity rises towards rigid corotation at lower latitudes. The latter region then corresponds to the main auroral oval as in the original model of Cowley and Bunce (2001). It was shown, however, that discrete auroras should also form in the upward current region at the boundary between open and closed field lines, this then relating to the polar auroral emissions lying poleward of the main oval (taken together with additional possible diffuse emission from precipitating hot plasma produced by related outer magnetosphere dynamics). Indeed, the polar emissions often take the form of an inner patchy ring surrounding a dark central region (Pallier and Prangé, 2001; Grodent et al., 2003b; Nichols et al., 2007), the latter then corresponding to open field lines in this interpretation.

All of the above models assume that steady-state conditions prevail. However, a characteristic feature of the interplanetary medium at Jupiter's orbit is the extreme and rapid variations of plasma and field parameters that are due to the formation of corotating interaction regions (CIRs) in the heliosphere, and to the outflow of coronal mass ejections (CMEs) from the Sun (e.g., Gosling and Pizzo, 1999; Nichols et al., 2006). The associated variations in the solar wind dynamic pressure are sufficient to regularly produce changes in the size of the jovian magnetosphere by factors of at least two in linear dimension (e.g., Huddleston et al., 1998). Minimum subsolar magnetopause radii are typically $\sim 45 R_{J}$ for maximum solar wind dynamic pressures of a few tenths of a $\mathrm{nPa}$, while maximum subsolar radii are typically $\sim 90 R_{J}$ for minimum dynamic pressures of a few hundredths of a $\mathrm{nPa}$ (Huddleston et al., 1998; Nichols et al., 2006). The expected effect of these changes on jovian auroral emissions within the above scenario was first discussed by Southwood and Kivelson (2001) and Cowley and Bunce (2001), who noted that the basic effect should be an anti-correlation of the intensity of the main oval with the solar wind dynamic pressure. This follows simply from the fact that a compression of the magnetosphere by an increase in the solar wind dynamic pressure will increase the angular velocity of the magnetospheric plasma via conservation of angular momentum (at least transiently), and will hence reduce the strength of the coupling currents and the intensity of the aurora. Similarly, an expansion of the magnetosphere due to a reduction in solar wind dynamic pressure will reduce the angular velocity of the magnetospheric plasma, hence increasing the strength of the coupling currents and the intensity of the aurora. The above authors also noted, however, that severe magnetospheric compressions could also induce transient super-corotation of the plasma in the outer regions of Jupiter's magnetosphere, evidence for which has been presented by Hanlon et al. (2004) in joint Galileo and Cassini spacecraft data. Middle magnetosphere super-corotation would lead to a reversal in the sense of the magnetosphere-ionosphere coupling current circuit, such that the usual region of main oval emission would become aurorally "dark", associated with downward-directed 
currents, while a new "main oval" might then form in the upward-directed closure current somewhere in the poleward region.

Simple axisymmetric middle magnetosphere models which demonstrate and quantify these effects were subsequently presented by Cowley and Bunce (2003a, b), showing, for example, that transient super-corotation can be excited by a sudden compression of the magnetosphere from $\sim 70$ to $\sim 45 R_{J}$. However, these studies only modelled the response of the middle magnetosphere, so that the issue of the formation of a new poleward oval could not be quantitatively investigated. They also assumed that the flow in the upper neutral atmosphere responds promptly to the effects of changing ion drag, while Gong and Hill (2005) have emphasised that current-reversal and auroral re-brightening could be enhanced significantly if the upper atmospheric flow responds only slowly to the changing plasma flow, since it is the plasma flow relative to the upper atmosphere flow that determines the instantaneous sense and magnitude of the coupling current system. With regard to the sub-corotating steady state that is eventually re-established following such transient responses, Cowley and Bunce (2003b) demonstrated that the intensity of the re-formed main oval should be positively correlated with system size, and hence anti-correlated with solar wind dynamic pressure, since plasma corotation is easier to enforce when the magnetosphere is compressed than when it is expanded, with a consequent weakening of the current system and auroras in the former case compared with the latter.

A rather complex auroral response to changes in solar wind dynamic pressure is thus expected on the basis of the above discussion, the modelling of which on a global basis is the principal topic of this paper. Relevant observations are, however, very sparse. Gurnett et al. (2002) and Pryor et al. (2005) have reported transient correlated increases in jovian UV and hectometric radio emissions during the Cassini Jupiter fly-by in December 2000-January 2001, that were associated with intervals of high dynamic pressure and field strength in the solar wind, apparently contrary to expectations based on the above discussion. It is notable, however, that the enhanced emissions occur for much shorter intervals than the interplanetary effects, a few hours compared with a few days. Gurnett et al. (2002) emphasised an association with the leading compressive shock of the related CIR/CME structures, but subsequent timing studies by Nichols et al. (2007) indicate that the brightenings are not specifically associated with the compression onsets. Instead, the events generally occur more centrally within the CIR/CME compression regions (which are characterised by strong variability), or even towards their end. It should also be noted that the Cassini UV data on which these studies are based are spatially unresolved, so that the auroral component in which the increases occurred remains unknown. Only one instance is currently known of an apparently related phenomenon observed in spatially resolved HST-STIS UV images, this also being observed during the Cassini Jupiter fly-by interval (Grodent et al., 2003a; Nichols et al., 2007). The relevant images show a main oval brightened by factors of two or three which is not significantly displaced in latitude compared with the usual reference oval within $\sim 1^{\circ}-2^{\circ}$ uncertainties, together with brightened extended patchy polar emissions. Concurrent interplanetary data obtained by Cassini show that this brightening also occurred during a solar wind compression interval of rapidly-varying properties, but timing uncertainties render it unclear whether the brightened oval is associated in detail with a modest (subsolar) magnetospheric compression from $\sim 55$ to $\sim 45 R_{J}$, or with a subsequent transient expansion to $\sim 75 R_{J}$ (Nichols et al., 2007). The status of observational information on these transient auroral phenomena and their relation to the solar wind is thus highly uncertain at present, and remains a central topic for future studies. This relative uncertainty further motivates the theoretical modelling work presented here.

\section{Theoretical model}

\subsection{Time scales and approximations}

It is evident that detailed calculation of the variations in magnetic field and plasma flow that take place in Jupiter's environment during substantial solar wind-induced compressions and expansions of the magnetosphere represents a considerable theoretical challenge. However, consideration of the various time scales involved suggests a number of approximations can be made that result in a much more tractable problem. The relevant time scales were considered in some detail by Cowley and Bunce (2003a) following earlier discussions by Nishida and Watanabe (1981) and Vasyliunas (1994), and will now be briefly reviewed since they also form the basis of the approximations employed here.

The basic time scale against which others must be compared is that of the magnetospheric compression or expansion itself. Clearly for sufficiently slow variations in solar wind parameters this is set by the solar wind time scale on which the dynamic pressure changes. However, we are particularly interested here in the step-like dynamic pressure changes associated with CIR- or CME-related interplanetary shocks, for which the time scale of the subsequent magnetospheric change will be a minimum. In this case the magnetopause boundary will move in or out at a speed which is some reasonable fraction of the solar wind speed, depending on the magnitude of the dynamic pressure jump, such that for boundary displacements of a few tens of $R_{J}$, the time scales involved will be $\sim 2 \mathrm{~h}$ (Cowley and Bunce, 2003a). On such time scales the modified interplanetary medium will also have engulfed much of the "forward" part of the magnetosphere. Solar wind changes taking place on significantly longer time scales will then produce correspondingly extended changes in magnetospheric configuration. 
As the magnetosphere expands or contracts under the action of the solar wind, the angular velocity of the nearequatorial plasma will also change due to conservation of angular momentum, as outlined in Sect. 1. This change will then be communicated along the field lines to the ionosphere at the Alfvén speed, the time scale for which forms the second quantity of interest. Estimates of the Alfvén speed outside the equatorial plasma sheet based on spacecraft data yield values in excess of $\sim 10^{4} \mathrm{~km} \mathrm{~s}^{-1}$, such that the propagation delays throughout the region of closed field lines are typically of order a few minutes. Such values are thus much shorter than even the minimum compression or expansion time scales. We therefore conclude that the magnetosphere and ionosphere remain in close communication throughout such intervals, with an essentially common angular velocity occurring along each field line between the equator and the ionosphere.

When the angular velocity of the plasma differs from that of the neutral atmosphere, a torque is imposed at the feet of the field lines in the ionosphere due to ion-neutral collisions in the Pedersen-conducting layer. This torque acts to return the plasma angular velocity towards that of the neutral atmosphere. The third time scale of interest is then the magnetosphere-ionosphere coupling time scale on which such angular momentum exchange takes place, which governs whether or not significant change in plasma angular momentum occurs on the expansion-compression time scale. The values for this time scale obtained by Cowley and Bunce (2003a) vary somewhat with radial distance, but are typically $\sim 5-20 \mathrm{~h}$ at equatorial distances beyond $\sim 20 R_{J}$. These time scales are thus significantly longer than the minimum time scale for magnetospheric compressions and expansions $(\sim 2 \mathrm{~h})$, thus indicating that for sufficiently rapid changes in solar wind properties leading to sufficiently rapid magnetospheric excursions, the plasma angular momentum is approximately conserved. A "rapid" change in solar wind dynamic pressure is thus one which takes place on time scales of $\sim 2-3 \mathrm{~h}$ or less in the planet's rest frame, leading to a $\sim 2-3 \mathrm{~h}$ magnetospheric response in which plasma angular momentum is approximately conserved. Conservation of plasma angular momentum thus forms the basis of our analysis of the transient magnetospheric response to such rapid changes in solar wind dynamic pressure.

The magnetosphere-ionosphere coupling time scale also forms an element of our estimate of the time scale on which steady-state conditions are resumed following such excursions. However, full resumption of steady-state conditions also requires the re-establishment of steady-state radial diffusive outflow of iogenic plasma through the equatorial plasma sheet. The time scale for this outflow can be estimated from consideration of the mass content and mass flux through the plasma sheet. On this basis Cowley and Bunce (2003a) indicate a time scale of $\sim 2$ days for outflow from $\sim 15 R_{J}$ to the outer edge of the middle magnetosphere at $\sim 50 R_{J}$, such that the plasma undertakes less than $\sim 5$ rotations about the planet during this transport. This fifth time scale is thus roughly comparable with, if a little longer than, the magnetosphereionosphere coupling time in the outer parts of the middle magnetosphere. Overall, therefore, we may expect that following significant compressions or expansions of the magnetosphere, steady state sub-corotational conditions will be resumed over intervals of $\sim 1-2$ days.

The sixth and final time scale of interest is the response time of the flow in the upper neutral atmosphere in the Pedersen layer, as discussed recently by Gong and Hill (2005). Under steady-state conditions the upper atmosphere will subcorotate relative to the planet at an angular velocity that is intermediate between that of the planet and the plasma. This is due to the action of ion-neutral collisions, which produces a torque on the neutral atmosphere that is equal and opposite to that on the plasma. When the plasma angular velocity changes during a compression or expansion event, a corresponding change will thus be induced in the neutral atmosphere, and we are interested in the time scale on which this change takes place. Gong and Hill (2005) estimated that this time scale is very long, $\sim 2$ days, based on the model of Huang and Hill (1989) in which the angular momentum of the upper neutral atmosphere is maintained by viscous transport from below. In this case, the neutral atmospheric flow will essentially not respond at all during rapid compressions and expansions of the magnetosphere (on time scales of $\sim 2-$ $3 \mathrm{~h}$ ), but will instead change slowly on a time scale that is similar to the overall time scale on which steady-state conditions are resumed. On the other hand, atmospheric modelling studies presented by Millward et al. (2005) indicate a much more prompt atmospheric response to changing plasma velocities in the Pedersen layer, on time scales of a few tens of minutes, mediated principally through horizontal transport of atmospheric angular momentum. Such time scales are then rather shorter than even the minimum compression and expansion time scales, indicating that the neutral atmospheric flow in the Pedersen layer responds quickly to changes in the plasma angular velocity. In effect, this was the assumption employed in the previous modelling studies by Cowley and Bunce (2003a, b). On the basis of the modelling results of Millward et al. (2005) this "prompt" response assumption will also be used as the baseline here, though due to existing uncertainties, results will also be presented for the opposite assumption of an "unresponsive" atmospheric flow on the rapid compression-expansion time scale.

\subsection{Axi-symmetric assumption}

A second important area of approximation concerns the geometry of the modelled system, which is assumed throughout to be axi-symmetric. This is evidently a reasonable approximation in the inner and middle magnetosphere regions of closed field lines, but clearly ignores major features of daynight asymmetry in the outer parts of the system. The specific model of the equatorial field and its response to solar 
wind dynamic pressure changes proposed in Sect. 2.4 below is intended principally to represent the dayside parts of the system bounded by the magnetopause. Nevertheless, related compressive and expansive effects due to solar wind-induced changes in system size will occur at all local times.

As a consequence of the axi-symmetric assumption the only flows that are described in the model are azimuthal flows, quantified by the plasma angular velocity on given flux shells. These are nevertheless expected to be the principal flows within the system. Consequently, the local time effects associated with the Dungey cycle of solar wind-induced outer magnetosphere flow (e.g., Cowley et al., 2003b) are not included, though the model does contain a representation of a semi-stagnant region of open field lines at the highest magnetic latitudes. Similarly, flow asymmetries associated with the Vasyliunas cycle of iogenic plasma mass loss through down-tail plasmoid ejection (Vasyliunas, 1983) are also not included, though again the model does include an "outer magnetosphere" region, based principally on dayside data, that is suggested to be associated mainly with this transport cycle. Although our axi-symmetric model is therefore clearly rather simplistic in nature, it does attempt to represent major structural features of the jovian system. In describing the large-scale changes in plasma angular velocity that occur during magnetospheric compressions and expansions we also suggest that it captures much of the essential physics while retaining considerable simplification of analysis.

\subsection{Ionospheric field, flow, and currents}

Analysis of the model begins here with a description of the field, flows, and currents in the jovian polar ionosphere mapping to the middle and outer parts of the magnetosphere. This region lies within $\sim 20^{\circ}$ co-latitude of the pole, while at lower latitudes the plasma near-rigidly corotates with the planet, except on field lines mapping near the inner moons whose localised effects on the flow are not included in this study. According to the modelling results of Millward et al. (2002), the jovian Pedersen conducting layer of the ionosphere lies $\sim 500 \mathrm{~km}$ above the atmospheric 1 bar pressure level, which itself has a radius at the pole of $66854 \mathrm{~km}$. Consequently, here we take the polar Pedersen layer to lie at a radius $R_{i}$ of $67350 \mathrm{~km}$. Since the co-latitude range about the pole considered here is modest, we employ this value over the whole of the ionospheric region of interest. Turning now to the magnetic field in this layer, according to the VIP4 magnetic model derived by Connerney et al. (1998), this varies significantly and comparably in latitude and longitude in the polar region of interest, and also between hemispheres. Since the longitude variations clearly cannot be represented in an axi-symmetric model, here we choose to employ the simplest possible ionospheric magnetic model, consisting of a radial field of fixed strength $B_{i}$. The model value of $B_{i}$ is taken to be $1.1 \times 10^{-3} \mathrm{~T}$, which is overall representative of the VIP4 field at polar latitudes. These approximations and values are identical to those employed in the steady-state axi-symmetric Jupiter model of Cowley et al. (2005). Field lines from the ionosphere are then mapped into the equatorial plane through consideration of magnetic flux conservation. Specifically we use a flux function $F$ related to the field components by $\boldsymbol{B}=(1 / \rho) \nabla F \times \hat{\boldsymbol{\varphi}}$, where $\rho$ is the perpendicular distance from the magnetic axis and $\hat{\varphi}$ is the unit vector in the direction of increasing azimuth around the axis. The properties of $F$ are such that the surface $F=$ const defines a shell of field lines around the magnetic axis, with the total amount of magnetic flux lying between shells $F$ and $F+d F$ being $d \Phi=2 \pi d F$. With the above assumptions concerning the ionospheric field, it is easy to show that the flux function as a function of co-latitude $\theta_{i}$ in the ionosphere is given by

$F\left(\theta_{i}\right)=R_{i}^{2} B_{i}\left(1-\cos \theta_{i}\right)$,

where the arbitrary zero of $F$ has been taken to lie at the pole, $\theta_{i}=0$.

We now consider the ionospheric currents that flow as a consequence of magnetosphere-ionosphere coupling. We suppose that the plasma angular velocity on a particular flux shell mapping to the ionosphere at co-latitude $\theta_{i}$ is $\omega\left(\theta_{i}\right)$, and that the corresponding neutral atmospheric angular velocity at this co-latitude is $\Omega_{J}^{*}\left(\theta_{i}\right)$, modified from the planetary angular velocity $\Omega_{J}$ (equal to $1.76 \times 10^{-4} \mathrm{rad} \mathrm{s}^{-1}$ ) due to the ion-neutral collisional torque mentioned in Sect. 2.1. Then the co-latitudinal ionospheric Pedersen current intensity driven by the electric field in the neutral atmosphere rest frame, positive equatorward when $\Omega_{J}^{*}>\omega$, is given by

$i_{P}\left(\theta_{i}\right)=\Sigma_{P} \rho_{i}\left(\Omega_{J}^{*}-\omega\right) B_{i}$,

where $\Sigma_{P}$ is the height-integrated ionospheric Pedersen conductance, and $\rho_{i}=R_{i} \sin \theta_{i}$ is the perpendicular distance from the magnetic axis. The total Pedersen current flowing at colatitude $\theta_{i}$, integrated in azimuth around the axis, is then

$I_{P}\left(\theta_{i}\right)=2 \pi \rho_{i} i_{P}\left(\theta_{i}\right)=2 \pi \Sigma_{P} \rho_{i}^{2}\left(\Omega_{J}^{*}-\omega\right) B_{i}$.

Variation of $I_{P}$ with $\theta_{i}$ then requires field-aligned currents to flow between the ionosphere and magnetosphere to maintain current continuity, the current density of which just above the ionosphere is given by

$$
j_{\| i}\left(\theta_{i}\right)=-\frac{1}{2 \pi R_{i}^{2} \sin \theta_{i}} \frac{d I_{P}}{d \theta_{i}},
$$

where the sign is appropriate to the Northern Hemisphere where the magnetic field points outward from the planet. We also note that Hall currents are driven in the jovian ionosphere by the plasma-neutral relative flow, directed eastward for sub-corotational flow. However, in our axi-symmetric model these close wholly in the ionosphere, and do not contribute to the field-aligned currents. Calculation of the auroral effects associated with regions of upward-directed fieldaligned current will be considered in Sect. 2.6 below, once further details of the model have been described. 
We now discuss the model assumptions concerning the behaviour of the neutral atmospheric angular velocity $\Omega_{J}^{*}\left(\theta_{i}\right)$. During steady-state conditions we assume that the subcorotation of the neutral atmosphere is some fixed fraction $k$ of the sub-corotation of the plasma, that is

$\Omega_{J}^{*}\left(\theta_{i}\right)=\Omega_{J}-k\left(\Omega_{J}-\omega\left(\theta_{i}\right)\right)$,

where $k \approx 0.5$ according to the modelling study presented by Millward et al. (2005). Substitution of Eq. (5) into Eq. (1) for the Pedersen current intensity then shows that $\Omega_{J}^{*}\left(\theta_{i}\right)$ may be replaced in that formula by the full planetary angular velocity $\Omega_{J}$, provided that $\Sigma_{P}$ is also replaced by the reduced "effective" Pedersen conductance $\Sigma_{P}^{*}=(1-k) \Sigma_{P}$. This "effective" conductance formulation has been employed in many previous studies, including that of Cowley and Bunce (2003a, b), thus in effect assuming that the neutral atmospheric wind responds to the plasma flow on time scales that are short compared with the plasma time scales of interest. The recent modelling results presented by Millward et al. (2005) suggest that this is indeed the case as indicated in Sect. 2.1 above, such that this will be taken as the baseline assumption in the results derived in Sect. 3. The contrary view of Gong and Hill (2005) that the relevant atmospheric response time is very long requires instead that $\Omega_{J}^{*}\left(\theta_{i}\right)$ remains essentially fixed during rapid plasma flow changes, determined by the plasma angular velocity profile in the pre-existing steady state, and only responds to the changed plasma flows as steady-state conditions are reestablished on time scales of $\sim 1-2$ days. Due to the present uncertainties, results based on this alternate assumption will also be presented for comparison. With regard to the value of the Pedersen conductivity, this is a parameter that is not well-determined at the present time. Here, however, we employ the value $\Sigma_{P}=0.4$ mho, such that with $k=0.5$ we also have $\Sigma_{P}^{*}=0.2$ mho. As discussed previously by Cowley et al. (2005) (see their Sect. 3.2), the latter value is found to produce reasonably realistic results when employed in magnetosphere-ionosphere coupling calculations.

The final ionospheric topic to be discussed here is the power extracted from planetary rotation by magnetosphereionosphere coupling, which is fed both into magnetospheric rotation and into upper atmospheric heating. The total power per unit area of ionosphere extracted from planetary rotation is just $p=\Omega_{J} \tau$ (e.g., Hill, 2001), where $\tau=\rho_{i} i_{P} B_{i}$ is the torque per unit area associated with the height-integrated $j \times B$ force. Of this total, the amount transferred to the magnetosphere is

$p_{M}\left(\theta_{i}\right)=\omega \tau=\omega \rho_{i} i_{P} B_{i}$.

The remainder is dissipated as heat in the upper atmosphere at the rate

$p_{A}\left(\theta_{i}\right)=\left(\Omega_{J}-\omega\right) \tau=\left(\Omega_{J}-\omega\right) \rho_{i} i_{P} B_{i}$,

consisting of both direct "Joule heating" in the Pedersen layer, and "ion drag" heating associated with sub-corotation of the neutral atmosphere that is viscously dissipated to heat at some atmospheric level (Smith et al., 2005).

\subsection{Model of the equatorial field and its response to varia- tions in system size}

We next consider the description of the equatorial magnetospheric magnetic field and its variations during compressions and expansions of the system. This is a critical component of the model, since it is the radial motion of the field lines during solar wind-induced excursions that determines the variation of the angular velocity of the equatorial plasma, and hence of the coupling currents and auroral precipitation. As indicated above, the magnetic model is intended primarily to reflect dayside conditions in which the closed field line regime is bounded by the magnetopause at an equatorial radius of $R_{M P}$ (a constant in the axi-symmetric approximation). Inside the magnetopause the model equatorial field is then taken to consist of two components. The first represents the middle magnetosphere field from an equatorial radial distance of $\sim 15 R_{J}$ where rigid corotation begins to break down, to an outer radius of $R_{M M}$. The region between $R_{M M}$ and $R_{M P}$ then represents the outer magnetosphere layer. Physically, the middle magnetosphere corresponds to the plasma disk region where cool iogenic plasma diffuses outward over a few jovian rotations as described in Sect. 2.1. Its field is characterised by strong radial current sheet components which reverse in sense about the equatorial plane, together with a weaker field that threads southward through the equator. The field in the outer magnetosphere, on the other hand, is dominated by southward components comparable in strength to the radial field outside the current sheet in the outer middle magnetosphere (e.g., Acuña et al., 1983), with no indication of an equatorial current layer. This region is suggested to correspond physically to the outer layer of mass-reduced flux tubes that have taken part in the Vasyliunas- and Dungey-cycles of flow mentioned in Sect. 2.2 above (Cowley et al., 1996; Kivelson and Southwood, 2006). Overall, therefore, the southwarddirected equatorial field in our model decreases in magnitude with increasing radial distance to small values in the outer middle magnetosphere, and then increases again within the outer magnetospheric layer bounded by the magnetopause. We note that Kivelson and Southwood (2006) have suggested that rapid mixing of the middle and outer magnetospheric flux tubes removes this dayside layering as the plasma rotates towards dusk, thus resulting in the observed thickening of the plasma sheet in that sector. This effect is not included in the axi-symmetric model constructed here.

Our approach to modelling the equatorial field and its variations is first to define a "baseline" model corresponding to a particular magnetosphere radius, and then to add or subtract a uniform field to model the effect of compressions or expansions, this representing the field due to the modified magnetopause currents then flowing. The "baseline" 
equatorial field representing the middle magnetosphere is based on Voyager-1 magnetic field data derived by Khurana and Kivelson (1993), as employed in previous modelling studies e.g. by Cowley and Bunce (2001, 2003a, b). This field is given by

$B_{M M 0}\left(r_{e}\right)=A\left(\frac{R_{J}}{r_{e}}\right)^{m}$,

where $A=5.4 \times 10^{4} \mathrm{nT}, \quad m=2.71, \quad$ and (as above) $R_{J}=71,373 \mathrm{~km}$. The strength of the field thus falls from a value of $\sim 16 \mathrm{nT}$ at a radial distance of $20 R_{J}$, to e.g. $\sim 0.7 \mathrm{nT}$ at $65 R_{J}$. Examination of concurrent Voyager-2 interplanetary data presented by Huddleston et al. (1998) shows that this field corresponds to conditions of relatively low solar wind dynamic pressure, $\sim 0.01-0.05 \mathrm{nPa}$, which thus corresponds to a relatively expanded magnetosphere. According to the analysis presented by Huddleston et al. (1998), the subsolar radius of Jupiter's magnetosphere is related to the solar wind dynamic pressure $p_{S W}$ by

$R_{M P} \approx\left[\frac{35.5}{\left(p_{S W}(n P a)\right)^{0.22}}\right] R_{J}$,

such that the above range of pressures corresponds to subsolar magnetopause radii between $\sim 70$ and $\sim 100 R_{J}$. Here we therefore take the middle magnetosphere field given by Eq. (8) to be associated with a "baseline" sub-solar magnetopause radius of $R_{M P 0}=85 R_{J}$ (corresponding to $\left.p_{S W} \approx 0.02 \mathrm{nPa}\right)$. Typically, the equatorial width of the dayside outer magnetosphere layer is $\sim 10-20 R_{J}$, depending on the size of the system (e.g., Acuña et al., 1983). In the "baseline" model we thus take the middle magnetosphere field to be given by Eq. (8) to a radial distance of $R_{M M 0}=65 R_{J}$, with the outer magnetosphere layer lying between 65 and $85 R_{J}$ beyond. The equatorial field strength in the outer magnetosphere layer is taken to be a constant whose value is set in accordance with observations, typically between $\sim 5$ and $\sim 15 \mathrm{nT}$ depending on the size of the system according to Acuña et al. (1983). Here we have taken the amount of magnetic flux threading the (axisymmetric) outer magnetosphere layer between $R_{M M O}$ and $R_{M P} 0$ to be $\Phi_{O M}=350 \mathrm{GWb}$, such that the "baseline" field strength in the layer is

$B_{O M 0}=\frac{\Phi_{O M}}{\pi\left(R_{M P 0}^{2}-R_{M M 0}^{2}\right)} \approx 7.3 \mathrm{nT}$.

The equatorial field strength therefore increases by about an order of magnitude across the boundary between the middle and outer magnetosphere regions at $65 R_{J}$ in this model. For comparison, the amount of magnetic flux threading the equatorial plane between 15 and $65 R_{J}$ in the middle magnetosphere current sheet region is $\sim 230 \mathrm{GWb}$.

As indicated above, in order to represent the effect of a compression of the system due to an increase in the solar wind dynamic pressure, we then add a uniform southward field $\Delta B$ to the "baseline" model. Negative values of $\Delta B$ which reduce the southward field then correspond to an expansion of the system beyond that of the "baseline". The perturbed boundary positions $R_{M M}$ and $R_{M P}$ corresponding to $\Delta B$ are then determined by the twin conditions that the amount of magnetic flux in both the outer and middle magnetosphere regions are individually preserved. The condition for the outer magnetosphere is thus simply

$\pi\left(R_{M P}^{2}-R_{M M}^{2}\right)\left(B_{O M 0}+\Delta B\right)=\Phi_{O M}$.

In the inner and middle magnetosphere we balance the increase in flux due to the addition of the field $\Delta B$ between the origin and $R_{M M}$, with the loss in flux of the "baseline" middle magnetosphere field due to the inward motion of the boundary from $R_{M M} 0$ to $R_{M M}$. We thus have

$$
\pi R_{M M}^{2} \Delta B=\frac{2 \pi A R_{J}^{2}}{(m-2)}\left[\left(\frac{R_{J}}{R_{M M}}\right)^{m-2}-\left(\frac{R_{J}}{R_{M M 0}}\right)^{m-2}\right] .
$$

Equations (11) and (12) thus provide two conditions joining the three quantities $\Delta B, R_{M M}$, and $R_{M P}$. Given any one of them, the other two can then be calculated (numerically in practice). Here we choose to specify the varying position of the magnetopause $R_{M P}$, approximately related to the solar wind dynamic pressure by Eq. (9), and then determine both $R_{M M}$ and $\Delta B$.

Results showing the model variation of $R_{M M}$ and $\Delta B$ with $R_{M P}$ are shown in Fig. 1. In Fig. 1a the solid line shows the distance of the outer boundary of the middle magnetosphere, $R_{M M}$, plotted versus $R_{M P}$ over the range of usual subsolar magnetopause distances between 40 and $100 R_{J}$. Its value increases from $\sim 25$ to $\sim 85 R_{J}$ over the range, with the dot on the line showing the value for the "baseline" model, i.e. $R_{M M}=R_{M M 0}=65 R_{J}$ when $R_{M P}=R_{M P 0}=85 R_{J}$. The dashed straight line of unit slope in the plot shows the corresponding equatorial magnetopause radius, so that the vertical distance between these two lines indicates the width of the outer magnetosphere layer, $\Delta R_{O M}=R_{M P}-R_{M M}$, also shown by the dot-dashed line in the lower part of figure. This remains close to $20 R_{J}$ throughout (the "baseline" value, also shown by the dot), though narrowing to $\sim 15 R_{J}$ for large compressions. The solid line in Fig. 1b similarly shows $\Delta B$ over the same range of magnetopause positions. The dashed line also shows the total field strength in the outer magnetosphere layer, $B_{O M}=B_{O M}+\Delta B$ with $B_{O M} \approx 7.3 \mathrm{nT}$ as given by Eq. (10), the dots again showing the values for the "baseline" model (zero for $\Delta B$ itself of course). It can be seen that for large compressions of the system the outer magnetosphere field increases to $\sim 15-20 \mathrm{nT}$, in line with observations (e.g., Acuña et al., 1983).

Using these results, in Fig. 2a we show the model southward equatorial fields, $B_{e}\left(r_{e}\right)$ versus $r_{e}$, for three subsolar magnetopause distances. The black line shows the "baseline" model given by Eq. (8) from small distances out to $65 R_{J}$, and by $B_{O M} 0$ given by Eq. (10) from $65 R_{J}$ to the magnetopause at $85 R_{J}$. This field corresponds to that of a relatively 


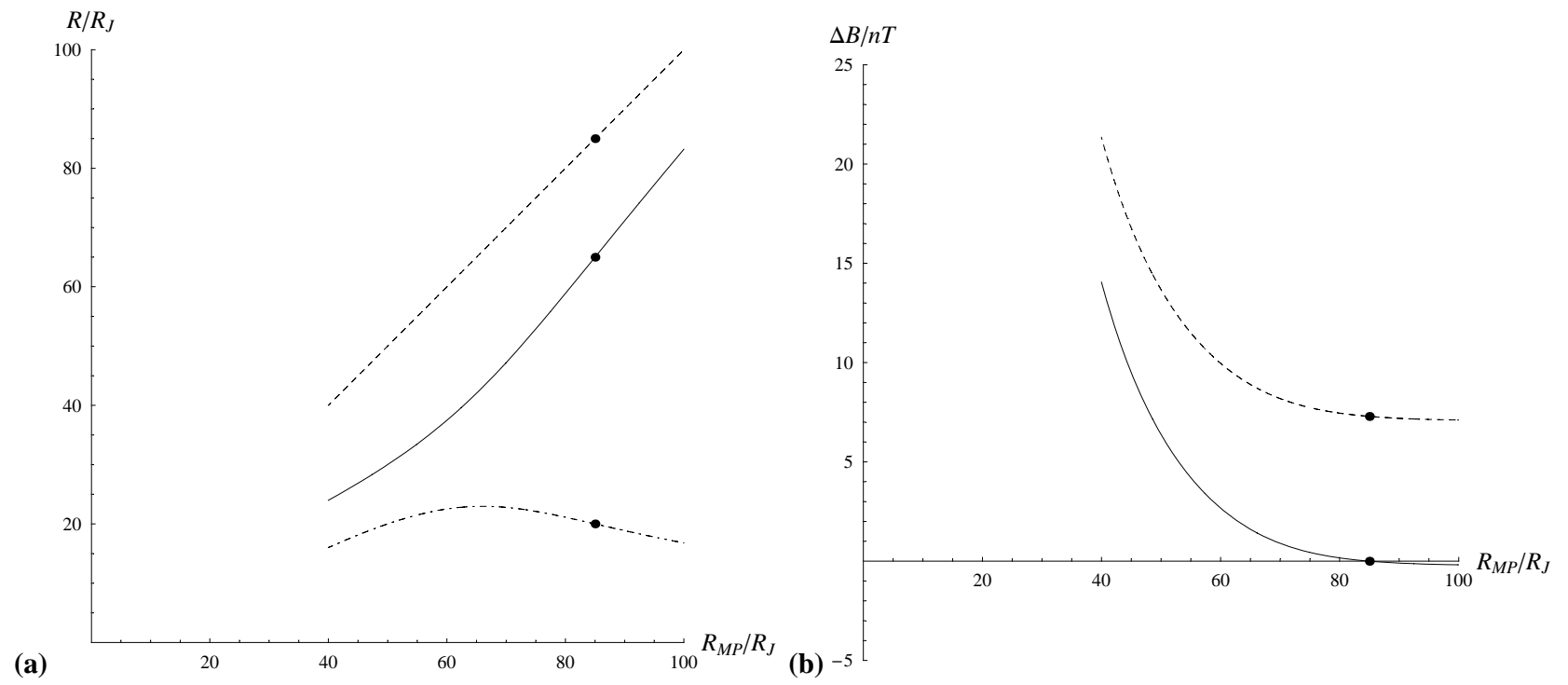

Fig. 1. (a) Plot showing the variation of the radius of the outer boundary of the middle magnetosphere region $R_{M M}$ (solid line) versus the magnetopause radius $R_{M P}$, obtained by simultaneous numerical solution of Eqs. (11) and (12), with parameter values given in the text. The radius is shown in units of Jupiter radii, $R_{J}$. The dashed straight line of unit slope shows the corresponding position of the magnetopause, so that the vertical distance between these lines corresponds to the width of the outer magnetosphere layer, $\Delta R_{O M}$. The latter width is also shown by the dot-dashed line in the lower part of the figure. The dots correspond to the values for the "baseline" model, for which $R_{M P}=85 R_{J}, R_{M M}=65 R_{J}$ and hence $\Delta R_{O M}=20 R_{J}$. (b) Plot showing the variation of the uniform perturbation field $\Delta B$ in $\mathrm{nT}$ (solid line) versus $R_{M P}$, which is added to the "baseline" model to represent compressions (positive values) and expansions (negative values) of the magnetosphere about the "baseline" value. The dashed line correspondingly shows the total model field in the outer magnetosphere, whose value is approximately $(\Delta B+7.29) \mathrm{nT}$ for the parameters employed here. The dots correspond to the values of the "baseline" model (zero for $\Delta B$ ).
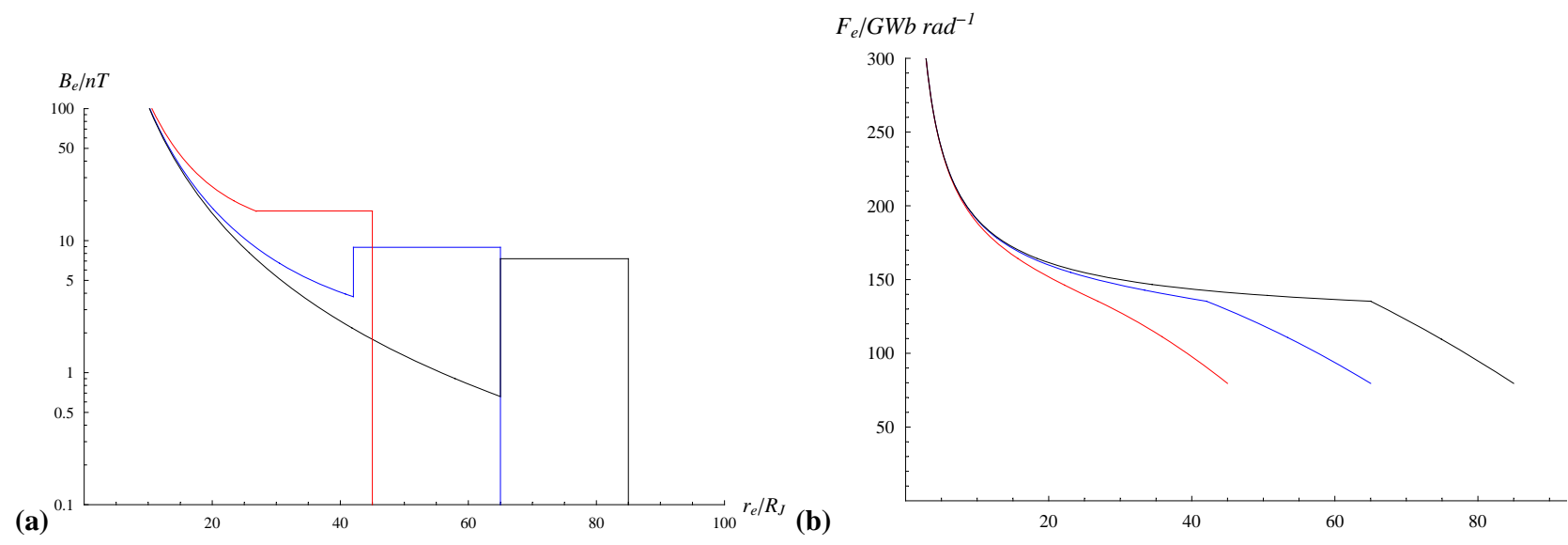

Fig. 2. (a) Plots of the model southward-directed field strength in Jupiter's equatorial plane, $B_{e}$ in nT, shown versus equatorial radial distance $r_{e}$ in $R_{J}$. The models shown correspond to subsolar magnetopause distances of 45 (red), 65 (blue), and $85 R_{J}$ (black), respectively. The black line thus corresponds to the "baseline" model, with the others derived from it according to the algorithm developed in Sect. 2.4. (b) Plots of the corresponding equatorial flux function $F_{e}$ in $\mathrm{GWb} \mathrm{rad}^{-1}$, using the same colour code. These were calculated from Eqs. (13) and (14) in the middle and outer magnetosphere regions, respectively.

expanded magnetosphere with a low solar wind dynamic pressure of $\sim 0.02 \mathrm{nPa}$ (roughly in line with the outer magnetosphere field pressure), as indicated above. The coloured lines then show the equatorial fields of more compressed systems according to the above algorithm. The blue line shows the field for an intermediate subsolar magnetopause at $65 R_{J}$, 
corresponding to a typical solar wind dynamic pressure of $\sim 0.06 \mathrm{nPa}$ according to Eq. (9). The red line similarly shows the field for a relatively compressed subsolar magnetopause at $45 R_{J}$, corresponding to a relatively high solar wind dynamic pressure of $\sim 0.3 \mathrm{nPa}$. In the latter case, the jump in equatorial field strength at the boundary between the middle and outer magnetosphere has essentially disappeared.

Having thus determined the magnetic fields in both the ionosphere and the equatorial plane, we can now establish the field line mapping between these regions. This is effected using conservation of magnetic flux via the flux function $F$, the value of which in the ionosphere, $F_{i}$, is given by Eq. (1). The value in the equatorial plane, $F_{e}$, is given by integration of $d F_{e} / d r_{e}=-r_{e} B_{e}\left(r_{e}\right)$, with the use of a suitable initial condition. As mentioned in Sect. 2.2, we assume that a central region of the polar ionospheric flux consists of open field lines mapping to the magnetic tail. The total amount of open flux in the model, $\Phi_{T}$, is taken to be $500 \mathrm{GWb}$, corresponding e.g. to a field of $\sim 1.5 \mathrm{nT}$ in a tail lobe of radius $\sim 200 R_{J}$. The value of the flux function at the boundary between open and closed field lines in the ionosphere is thus $F_{i}=\Phi_{T} / 2 \pi \approx 79.6 \mathrm{GWb} \mathrm{rad}^{-1}$, corresponding to a co-latitude of $\theta_{i M P} \approx 10.25^{\circ}$ in the ionosphere according to Eq. (1). This must therefore also correspond to the flux function at the magnetopause in the equatorial plane, independent of its position. Integrating $F_{e}$ inwards from the magnetopause then yields for the outer magnetosphere

$$
\begin{aligned}
F_{e O M}\left(r_{e}\right) & =\frac{\Phi_{T}}{2 \pi}+\frac{\left(B_{O M 0}+\Delta B\right)}{2}\left(R_{M P}^{2}-r_{e}^{2}\right) \\
& =\frac{\Phi_{T}}{2 \pi}+\frac{\Phi_{O M}}{2 \pi}\left(\frac{R_{M P}^{2}-r_{e}^{2}}{R_{M P}^{2}-R_{M M}^{2}}\right),
\end{aligned}
$$

where use has been made of Eq. (1) in the second form on the right hand side. Thus at the magnetopause $\left(r_{e}=R_{M P}\right)$ we have $F_{e} O M\left(R_{M P}\right)=\Phi_{T} / 2 \pi$ as just indicated, while at the inner boundary of the outer magnetosphere, at $r_{e}=R_{M M}$, we have $F_{e} O M\left(R_{M M}\right)=\left(\Phi_{T}+\Phi_{O M}\right) / 2 \pi$, with a model value of $\sim 135.3 \mathrm{GWb} \mathrm{rad}^{-1}$ since we also have $\Phi_{O M}=350 \mathrm{GWb}$ as indicated above. The boundary between the middle and outer magnetosphere at radial distance $R_{M M}$ thus maps in our model to a co-latitude in the ionosphere of $\theta_{i M M} \approx 13.37^{\circ}$ according to Eq. (1), also independent of the magnetopause position. Finally, in the middle magnetosphere region the flux function is given by

$$
\begin{aligned}
F_{e M M}\left(r_{e}\right)= & \frac{\left(\Phi_{T}+\Phi_{O M}\right)}{2 \pi}+\frac{A R_{J}^{2}}{(m-2)} \\
& {\left[\left(\frac{R_{J}}{r_{e}}\right)^{m-2}-\left(\frac{R_{J}}{R_{M M}}\right)^{m-2}\right] } \\
& +\frac{\Delta B}{2}\left(R_{M M}^{2}-r_{e}^{2}\right),
\end{aligned}
$$

which also has the value $F_{e}\left(R_{M M}\right)=\left(\Phi_{T}+\Phi_{O M}\right) / 2 \pi$ at $r_{e}=R_{M M}$, as required. The equatorial flux functions given by

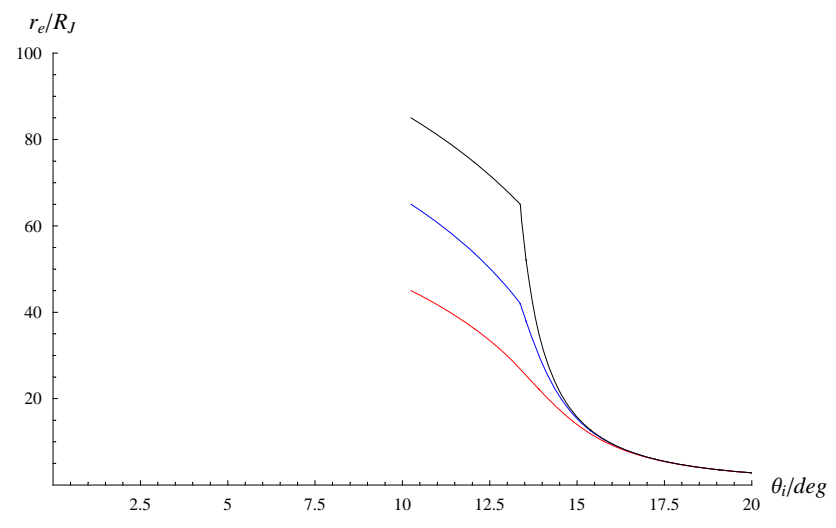

Fig. 3. Plot of the radial distance of field lines in the equatorial plane, $r_{e}$ in $R_{J}$, versus co-latitude in the ionosphere, obtained by conservation of magnetic flux between the ionosphere and the equator. The colour code is the same as for Fig. 2.

Eqs. (13) and (14) which correspond to the three equatorial field models shown in Fig. 2a are plotted versus $r_{e}$ in Fig. 2b, using the same colour code. The black line thus shows the flux function for the "baseline" model with a magnetopause boundary at $85 R_{J}$, while the blue and red lines correspond to magnetopause positions of 65 and $45 R_{J}$, respectively. Using the constancy of $F$ on flux shells, i.e. $F_{i}\left(\theta_{i}\right)=F_{e}\left(r_{e}\right)$, where $F_{i}$ is given by Eq. (1) and $F_{e}$ by Eqs. (13) and (14) in the outer and middle magnetospheres respectively, we can now use these results to map field lines between the ionosphere and equatorial plane. In Fig. 3 we thus show the equatorial radial distances of the field lines versus co-latitude in the ionosphere for the three equatorial field models shown in Fig. 2a, using the same colour code. Note again that the magnetopause positions at 45 (red), 65 (blue) and $85 R_{J}$ (black) map to a fixed ionospheric co-latitude at $\sim 10.25^{\circ}$, as do the boundaries between the outer and middle magnetosphere at a co-latitude of $\sim 13.37^{\circ}$. No values are shown poleward of $\sim 10.25^{\circ}$ since these field lines are open in model, and do not cross the equatorial plane.

\subsection{Plasma angular velocity model}

In the results to be presented in Sect. 3 it will be assumed that the jovian system is initially in a steady state with a fixed initial magnetopause radius, that it then undergoes a rapid compression or expansion to a new magnetopause radius due to a change in solar wind dynamic pressure on a time scale of a few hours or less, and that steady state conditions with the new radius are then resumed after an interval of $\sim 1-2$ days, assuming that the solar wind remains steady over this interval. In this section we outline the considerations on which the angular velocity profiles appropriate to these circumstances have been calculated, starting with the steady-state case.

The steady-state angular velocity profile in the middle magnetosphere is calculated by applying Newton's second 
law to the out-flowing iogenic plasma in the equatorial plasma sheet, assuming a constant radial mass flux $\dot{M}$ at distances between the Io torus $\left(\sim 6 R_{J}\right)$ and the outer boundary of the middle magnetosphere $R_{M M}$. This solution does not therefore apply in the outer magnetosphere region, taken to be a layer associated with the Vasyliunas- and Dungey-cycle flows, as mentioned above. Following the earlier analyses of Hill (1979) and Pontius (1997), the steady-state condition in the middle magnetosphere is thus such that the rate of change with radial distance of the outward flux of plasma angular momentum is equal to the torque per unit radial distance acting on the plasma due to the magnetosphere-ionosphere coupling current system. This condition leads directly to

$\dot{M} \frac{d}{d r_{e}}\left(r_{e}^{2} \omega\right)=2 \pi r_{e}^{2} i_{r} B_{e}\left(r_{e}\right)$,

where $i_{r}$ is the equatorial radial current intensity integrated through the thickness of the plasma sheet which is due to the ionosphere-magnetosphere coupling current circuit. From current continuity, this is related to the equatorward-directed ionospheric Pedersen current intensity $i_{P}$ at the feet of the same field lines by $r_{e} i_{r}=2 \rho_{i} i_{P}$, where $\rho_{i}$ is again the perpendicular distance of the field line feet in the ionosphere from the symmetry axis, and the factor of two accounts for both the Northern and Southern Hemispheres. Introducing this expression for $i_{r}$ into Eq. (15), with $i_{P}$ given by Eq. (2) and $\Omega_{J}^{*}$ in the steady state given by Eq. (5), then yields the Hill-Pontius equation for the steady-state angular velocity in the middle magnetosphere

$\dot{M} \frac{d}{d r_{e}}\left(r_{e}^{2} \omega\right)=4 \pi \Sigma_{P}^{*} B_{i} \rho_{i}^{2} r_{e} B_{e}\left(r_{e}\right)\left(\Omega_{J}-\omega\right)$,

where, as above, $\Sigma_{P}^{*}=(1-k) \Sigma_{P}$. We can also transform this equation to use ionospheric variables by noting that $d F_{e}=d F_{i}$ implies $r_{e} B_{e}\left(r_{e}\right) d r_{e}=-R_{i}^{2} B_{i} \sin \theta_{i} d \theta_{i}$, so we also have

$\dot{M} \frac{d}{d \theta_{i}}\left(r_{e}^{2}\left(\theta_{i}\right) \omega\right)=-4 \pi \Sigma_{P}^{*} B_{i}^{2} R_{i}^{4} \sin ^{3} \theta_{i}\left(\Omega_{J}-\omega\right)$

where $r_{e}\left(\theta_{i}\right)$ is the equatorial radial distance of the field lines at co-latitude $\theta_{i}$ in the ionosphere, as depicted in Fig. 3. This is a first order linear equation for $\omega$ whose solution may be obtained numerically with the aid of one initial condition. The condition required is that the plasma near-rigidly corotates with the planet at sufficiently small radial distances, corresponding to sufficiently large ionospheric co-latitudes. Here we have integrated Eq. (17) with the boundary condition that the plasma rigidly corotates (i.e. $\omega=\Omega_{J}$ ) at $\theta_{i}=25^{\circ}$, a co-latitude that corresponds to an equatorial radius in the innermost magnetosphere (see Fig. 3) where the Khurana and Kivelson (1993) field model given by Eq. (8) is no longer strictly applicable. However, previous analyses have shown that the solutions of Eq. (17) in the outer parts of the system where the Khurana and Kivelson (1993) model is valid are extremely insensitive to the inner boundary condition imposed (e.g., Nichols and Cowley, 2003), so we expect our steady-state solutions to be essentially independent of the details of this choice.

With regard to the outer magnetosphere layer, we have no simple theory with which to estimate the plasma angular velocity in the steady state and its dependence on system size. Observations in this region, however, suggest that the angular velocities generally do not differ greatly from those in the outer part of the middle magnetosphere, nor do they vary greatly with equatorial radial distance (e.g., Kane et al., 1995). Consequently, here we simply assume that the plasma angular velocity throughout the outer magnetosphere layer in the steady state is just equal to that at the outer edge of the middle magnetosphere region determined from integration of Eq. (17). Thus there is no discontinuity in the model plasma angular velocity at this boundary, though there will in general be a discontinuity in its slope.

With regard to the open field lines at highest latitudes, we assume that weak sub-corotation occurs in this region in accordance with the theory of Isbell et al. (1984), which gives the steady-state angular velocity of open flux tubes produced by the ionospheric torque on the field line feet as

$$
\left(\frac{\omega}{\Omega_{J}}\right)_{T}=\frac{\mu_{0} \Sigma_{P}^{*} V_{S W}}{1+\mu_{0} \Sigma_{P}^{*} V_{S W}}
$$

where $V_{S W}$ is the speed of the solar wind. With $\Sigma_{P}^{*}=0.2 \mathrm{mho}$ and e.g. $V_{S W}=450 \mathrm{~km} \mathrm{~s}^{-1}$, Eq. (18) gives $\left(\omega / \Omega_{J}\right)_{T} \approx 0.1$, such that this value (i.e. $10 \%$ of rigid corotation) has been employed for the open field region throughout the calculations presented here.

This then completes the description of the steady-state plasma angular velocity profiles. Examples are shown in Fig. 4 for the same three magnetic field models shown in Figs. 2 and 3, where we have also employed the same colour code. The system parameters are $\Sigma_{P}^{*}=0.2 \mathrm{mho}$ and $\dot{M}=1000 \mathrm{~kg} \mathrm{~s}^{-1}$, as have been used throughout these calculations. Figure $4 \mathrm{a}$ shows $\left(\omega / \Omega_{J}\right)$ versus radial distance on closed field lines in the equatorial plane. Rigid corotation is indicated by the horizontal dotted line at $\left(\omega / \Omega_{J}\right)=1$. In the middle magnetosphere region the angular velocities at a given radial distance do not depend strongly on which magnetic model is used, as found previously by Pontius (1997), but the profiles extend to significantly lower values at larger distances in the more extended cases. Consequently, in accordance with the above algorithm the angular velocities in the outer magnetospheric layer also fall as the system expands, thus mirroring both observations and the similar behaviour in the simple Jupiter models derived by Cowley et al. (2005). Figure $4 \mathrm{~b}$ shows the corresponding angular velocity profiles in the ionosphere. Here the transition between the fixed angular velocity $\left(\omega / \Omega_{J}\right)=0.1$ on open field lines and the consistently higher values in the outer magnetosphere have been smoothed using a hyperbolic tangent function, 

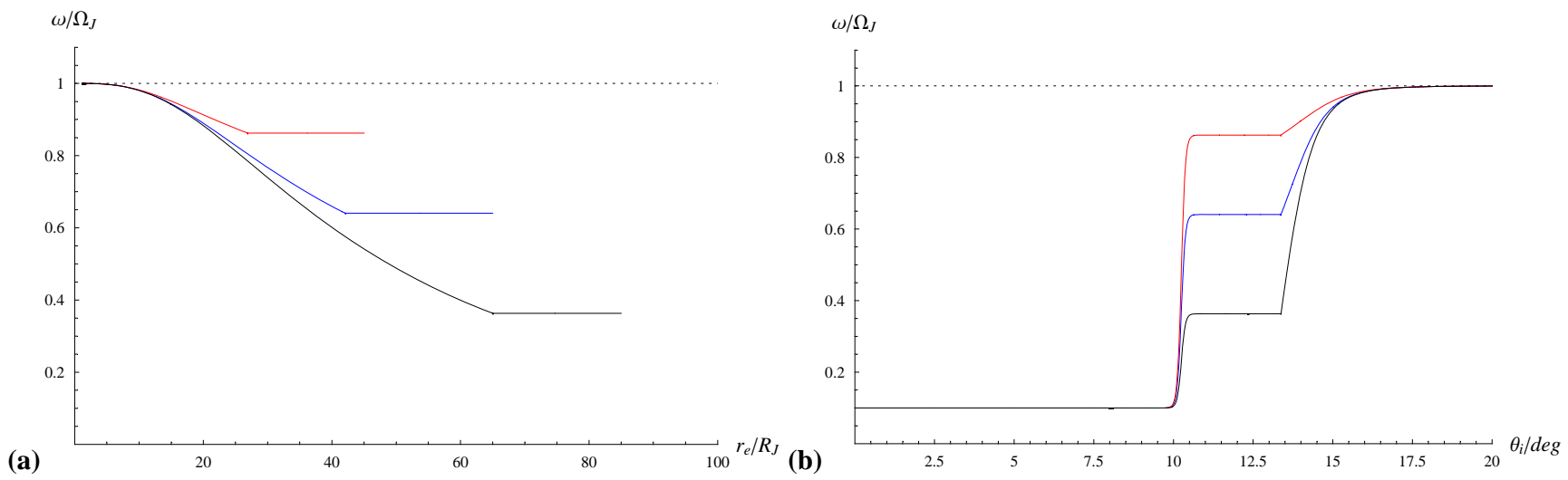

Fig. 4. (a) Steady-state normalised plasma angular velocity profiles $\left(\omega / \Omega_{J}\right)$ on closed field lines plotted versus equatorial radial distance $r_{e}$ for the three magnetic models shown in Fig. 2a. The black line thus shows the "baseline" model with the magnetopause at $85 R_{J}$, while the blue and red lines correspond to more compressed states with magnetopauses at 65 and $45 R_{J}$. The horizontal dotted line represents rigid corotation with the planet. (b) As in (a), but now plotted versus co-latitude in the ionosphere, and including the flow on open field lines. The transition between the outer and middle magnetosphere is evident at $\theta_{i} M M \approx 13.37^{\circ}$, as is the transition between open and closed field lines at $\theta_{i M P} \approx 10.25^{\circ}$, the latter being smoothed according to Eq. (19).

such that in the vicinity of the open-closed field line boundary we take

$$
\begin{aligned}
& \left(\frac{\omega}{\Omega_{J}}\right) \approx \frac{(1-\tau)}{2}\left(\frac{\omega}{\Omega_{J}}\right)_{T}+\frac{(1+\tau)}{2}\left(\frac{\omega}{\Omega_{J}}\right)_{O M}, \\
& \text { where } \tau=\tanh \left[\frac{\theta_{i}-\theta_{i M P}}{\Delta \theta_{i} M P}\right] .
\end{aligned}
$$

The width of the boundary region is thus determined by the parameter $\Delta \theta_{i M P}$, whose value has been taken to be $0.125^{\circ}$. The angular velocity transition between open and closed field lines is thus taken to occur over a latitude range of about $3 \Delta \theta_{i M P}=0.375^{\circ}$ in the ionosphere, this corresponding to a north-south spatial scale of $\sim 440 \mathrm{~km}$. This then sets the north-south scale of the field-aligned current flow occurring at the open-closed field line boundary in the ionosphere, due to the change in plasma angular velocity occurring there.

We now consider the calculation of the transient plasma angular velocity states that occur due to rapid compressions or expansions in the magnetosphere by the solar wind. Suppose that in the initial steady state the plasma angular velocity at a particular co-latitude in the ionosphere is $\omega^{\prime}\left(\theta_{i}\right)$ as determined above, and that the corresponding field lines reach to a radial distance of $r_{e}^{\prime}\left(\theta_{i}\right)$ in the equatorial plane. Suppose also that the magnetopause position then undergoes a rapid transition due to a change in the solar wind dynamic pressure, such that the field line at co-latitude $\theta_{i}$ now maps in the equatorial plane to a new radial distance $r_{e}\left(\theta_{i}\right)$ (corresponding, e.g. to a transition between the black, blue, or red curves in Fig. 3). Then assuming that the bulk of the magnetospheric plasma on a given field line is located near the equatorial plane, and that for a rapid transition the angular momentum of the plasma is approximately conserved in accordance with the discussion in Sect. 2.1, then the plasma angular velocity at co-latitude $\theta_{i}$ after the transition will be

$\omega\left(\theta_{i}\right)=\omega^{\prime}\left(\theta_{i}\right)\left(\frac{r_{e}^{\prime}\left(\theta_{i}\right)}{r_{e}\left(\theta_{i}\right)}\right)^{2}$

This is then the formula employed to transform the initial steady state plasma angular velocity profile into the transient state profile following rapid excursions of the magnetopause, the ratio $\left(r_{e}^{\prime}\left(\theta_{i}\right) / r_{e}\left(\theta_{i}\right)\right)$ being determined by the field line motion algorithm given in Sect. 2.4. Compressions and expansions should not, however, effect the rate at which open tail lobe field lines are twisted by the ionosphere, as described by the results of Isbell et al. (1984), so we take the angular velocity in the open field region to be unchanged during these processes. Results based on these considerations will be given in Sect. 3 below.

\subsection{Auroral parameters}

Once a plasma angular velocity profile has been determined, the horizontal ionospheric current follows from Eq. (2), augmented by choice of neutral atmospheric response model, and the field-aligned current density from Eq. (4). We then enquire whether downward-acceleration of magnetospheric electrons is required to carry the upward-directed currents, and if so, whether bright auroras are produced from the resulting precipitating electron energy flux.

The maximum field-aligned current density that can be carried by precipitating electrons (charge $e$, mass $m_{e}$ ) without field-aligned acceleration is

$j_{\| i 0}=e N\left(\frac{W_{t h}}{2 \pi m_{e}}\right)^{1 / 2}$, 
Table 1. Magnetospheric electron source parameters employed in auroral calculations.

\begin{tabular}{lllll}
\hline Parameter & Magnetosheath source & Outer magnetosphere source & Middle magnetosphere source \\
\hline $\begin{array}{l}\text { Electron density } N\left(\mathrm{~cm}^{-3}\right) \\
\begin{array}{l}\text { Electron thermal energy } \\
(\mathrm{keV})\end{array}\end{array} W_{t h}$ & 0.5 & 0.02 & 0.01 \\
$\begin{array}{l}\mathrm{Unaccelerated} \text { current density } \\
j_{\| \mid i 0}\left(\mu \mathrm{A} \mathrm{m}^{-2}\right)\end{array}$ & 0.095 & 0.25 & 2.5 \\
$\begin{array}{l}\text { Unaccelerated energy flux } \\
\left(\mathrm{mW} \mathrm{m}_{f 0}^{-2}\right)\end{array}$ & 0.0095 & 0.0085 & 0.013 \\
\hline
\end{tabular}

where we assumed that the magnetospheric source population is an isotropic Maxwellian of density $N$ and thermal energy $W_{t h}$ (equal to $k T$ where $T$ is the temperature and $k$ is Boltzmann's constant). We also assumed that the electron population has a full downward-going loss cone, and an empty upward-going loss cone. The corresponding precipitating electron energy flux is

$E_{f 0}=2 N W_{t h}\left(\frac{W_{t h}}{2 \pi m_{e}}\right)^{1 / 2}$.

If the upward-directed current density required by Eq. (4) is larger than $j_{\| i 0}$ given by Eq. (21), then a field-aligned voltage must occur which accelerates the electrons into the ionosphere. According to Knight's (1973) kinetic theory the minimum field-aligned voltage required is

$\Phi_{\|}=\frac{W_{t h}}{e}\left(\left(\frac{j_{\| i}}{j_{\| i 0}}\right)-1\right)$,

this value being appropriate if the "top" of the voltage drop is located well above the minimum radial distance

$$
\left(\frac{r_{\min }}{R_{i}}\right) \approx\left(\frac{j_{\| i}}{j_{\| i 0}}\right)^{1 / 3}
$$

where we have assumed that the field strength drops as the inverse cube of the distance along the polar field lines. Equation (23) also assumes that the voltage drop is sufficiently compact along the field lines that electrons do not mirror before experiencing the full field-aligned voltage. Following Lundin and Sandahl (1978), the enhanced precipitating electron energy flux corresponding to Eq. (23) is then

$E_{f}=\frac{E_{f 0}}{2}\left(\left(\frac{j_{\| i}}{j_{\| i 0}}\right)^{2}+1\right)$.

Here we will use Eqs. (23-25) to estimate the electron acceleration conditions and precipitating energy fluxes in regions of upward field-aligned current, using typical observed values of the magnetospheric electron source parameters. For simplicity, in the calculations presented below different fixed values will be employed in each of the three regions of the model, corresponding to the magnetosheath on open field lines (actually applied only in the region of upward fieldaligned current in the vicinity of the open-closed field line boundary), and the outer and middle magnetosphere regions on closed field lines. The parameter values are thus switched from one source population to the other across the region boundaries at $\theta_{i M P}$ and $\theta_{i M M}$ in the ionosphere. These values, together with the associated limiting current densities and energy fluxes given by Eqs. (21) and (22) are listed in Table 1 , as employed previously by Cowley et al. (2005). The middle magnetosphere values are based on Voyager electron data presented by Scudder et al. (1981), while the outer magnetosphere and magnetosheath values are based on Ulysses data presented by Phillips et al. (1993a, b). It can be seen that the limiting current densities in the middle and outer magnetosphere regions are both $\sim 0.01 \mu \mathrm{A} \mathrm{m}^{-2}$, while that of the magnetosheath source is an order of magnitude higher, $\sim 0.1 \mu \mathrm{A} \mathrm{m}^{-2}$.

The UV auroral output can be estimated from the precipitating electron energy flux. Auroras are excited by primary and secondary electron impact on atmospheric atomic and molecular hydrogen, resulting in the emission of $\sim 10 \mathrm{eV}$ UV photons corresponding to the atomic hydrogen Lyman alpha line and the molecular hydrogen Lyman and Werner bands. The energy efficiency of these processes is $\sim 15 \%$ (Waite et al., 1983; Rego et al., 1994), such that as a simple approximation we can assume that $1 \mathrm{~mW} \mathrm{~m}^{-2}$ of precipitating electron input yields $\sim 10 \mathrm{kR}$ of UV output (1 Rayleigh (R) corresponds to a photon source flux of $10^{10} \mathrm{~m}^{-2} \mathrm{~s}^{-1}$ ). Table 1 thus shows that precipitation from the unaccelerated populations alone would yield only sub-kR emissions, below the $\sim 1 \mathrm{kR}$ threshold of present detectability. For highenergy (e.g. $\sim 100 \mathrm{keV}$ ) primary accelerated electrons that penetrate more deeply into the atmosphere, the UV output may be somewhat reduced from these levels due to absorption by hydrocarbons overlying the emitting layer.

\section{Magnetospheric compression}

The model algorithms discussed in Sect. 2 describe the steady-state condition of the jovian magnetosphere for any 
chosen value of its radius, determined by the solar wind dynamic pressure, together with the transient states produced by rapid solar wind-induced excursions from any given radius to any other. In this section and that following we exemplify the properties of this model by presenting results for major compressions and expansions of the magnetosphere. Specifically, in this section we examine the results of a rapid compression of the model magnetosphere inwards from an initial radius of $85 R_{J}$, appropriate to a low-pressure solar wind rarefaction region, to 65 and to $45 R_{J}$, the latter corresponding to a high-pressure compression region. In Sect. 4 we then consider the effects of a rapid expansion back from $45 R_{J}$, to 65 and to $85 R_{J}$.

\subsection{Plasma angular velocity profiles}

In Fig. 5 we thus show results for the effect of rapid magnetospheric compression on the equatorial plasma angular velocity profile. The black solid line shows the initial steady-state system extending to a magnetopause at $85 R_{J}$, as in Fig. $4 \mathrm{a}$, thus corresponding to steady-state conditions with the "baseline" magnetic field model. The blue and red solid lines then show the transient angular velocity profiles produced by rapid compressions inward to 65 and to $45 R_{J}$, respectively, conserving angular momentum, while the blue and red dashed lines show the steady states to which these perturbed systems relax after 1-2 days (also as in Fig. 4a). It can be seen that a compression from 85 to $65 R_{J}$ is sufficient to raise the plasma angular velocities significantly toward rigid corotation throughout the closed field region, such that the angular velocity in the middle magnetosphere falls only to $\left(\omega / \Omega_{J}\right) \approx 0.85$ at its outer edge, while that in the outer magnetosphere is raised from $\sim 0.35$ to $\sim 0.7$. Further compression of the magnetopause to $45 R_{J}$ is then sufficient to induce significant super-corotation throughout the magnetosphere, reaching a peak of $\left(\omega / \Omega_{J}\right) \approx 2$ at the boundary between the middle and outer magnetosphere regions.

\subsection{Magnetosphere-ionosphere coupling current system}

\subsubsection{Results for the case of prompt atmospheric flow re- sponse}

We now consider the consequences of these angular velocity profiles for the model current systems. In this sub-section we assume that the neutral atmosphere responds promptly to the plasma angular velocity changes as discussed in Sect. 2.3 above, such that its angular velocity $\Omega_{J}^{*}$ is given by Eq. (5) with $k=0.5$. In other words, the departure of the neutral atmosphere from rigid corotation is taken always to be just half that of the plasma. To compute the currents the equatorial angular velocity is first transformed along the field lines into the ionosphere, resulting in the profiles shown in Fig. 6a. Note that the angular velocity on open field lines at highest latitudes is assumed to be unchanging. Introducing $\omega$ into

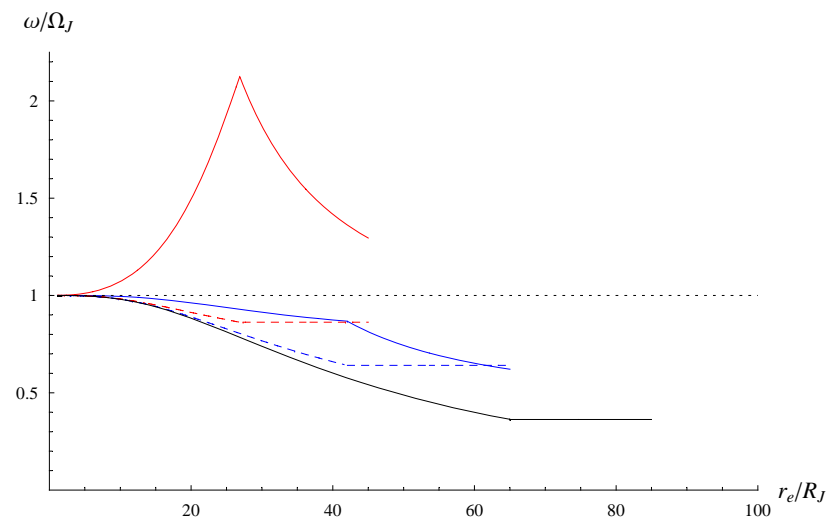

Fig. 5. Plots of the normalised plasma angular velocity shown versus equatorial radial distance for the case of solar wind compression of the magnetosphere. The black solid line shows the initial steady state profile with a magnetopause at $85 R_{J}$ and a boundary between the middle and outer magnetosphere at $65 R_{J}$ (the "baseline" model). The blue and red solid lines then show the transient profiles following a rapid inward compression of the magnetopause to 65 and $45 R_{J}$, respectively. The red and blue dashed lines show the steady states to which these profiles relax after a $\sim 1-2$ day interval. The horizontal dotted line corresponds to rigid plasma corotation with the planet.

Eq. (2), $\Omega_{J}^{*}$ given by Eq. (5) with $k=0.5$, and $\Sigma_{P}=0.4$ mho as indicated above (such that the "effective" conductivity is constant and equal to $0.2 \mathrm{mho}$ ), then yields the ionospheric Pedersen current intensity profiles shown in Fig. 6b. In the initial steady state, the current grows with the velocity of the plasma relative to the neutral atmosphere on open field lines, falls as the angular velocity increases on moving onto closed field lines, increases again in the outer magnetosphere, and then falls rapidly across the middle magnetosphere as the angular velocity increases towards rigid corotation. In the transient compressed states, the Pedersen current is significantly reduced throughout the closed field region for the compression to $65 R_{J}$ due to the increased plasma angular velocities closer to rigid corotation, while being reversed in sense throughout this region for the compression to $45 R_{J}$ due to plasma super-corotation. The corresponding total Pedersen current profiles given by Eq. (3) are shown in Fig. 6c. In the initial steady state the equatorward current peaks at $\sim 40 \mathrm{MA}$ at the boundary between the outer and middle magnetosphere regions, with a subsidiary maximum of $\sim 30 \mathrm{MA}$ at the openclosed field line boundary. The latter peak remains when the system is compressed inwards to $65 R_{J}$, but now the current on closed field lines is strongly reduced to form a shallow maximum at $\sim 15 \mathrm{MA}$ within the outer magnetosphere region, while falling continuously towards zero across the inner part of the outer magnetosphere and in the middle magnetosphere region. When the system is compressed to $45 R_{J}$, however, the currents are reversed to poleward everywhere on closed field lines, with a peak poleward-directed 


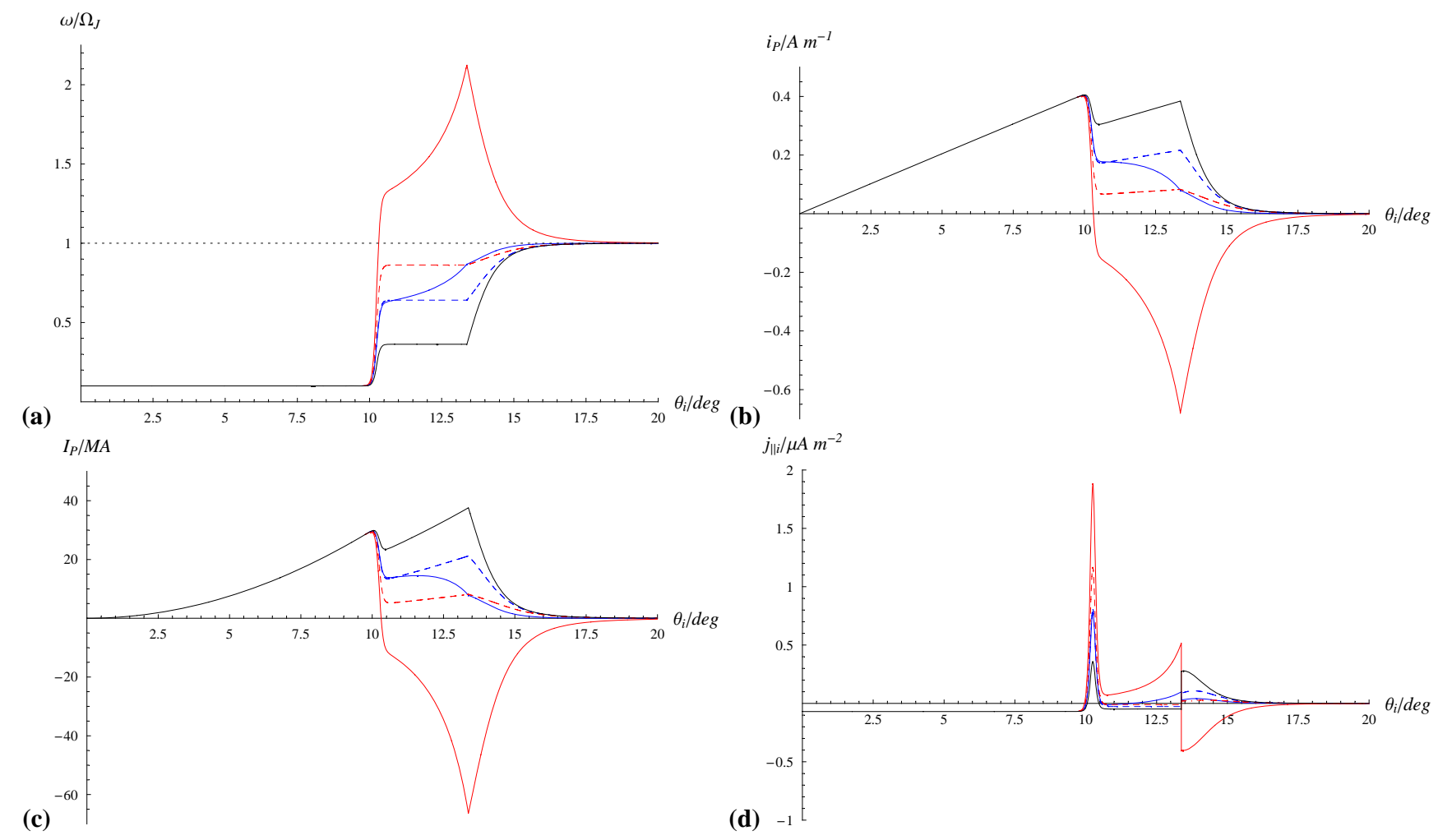

Fig. 6. Plasma angular velocity profiles and ionospheric currents plotted versus ionospheric co-latitude for compression of the magnetosphere from $85 R_{J}$ (black lines), to 65 (blue) and $45 R_{J}$ (red). The blue and red solid lines correspond to the transient states produced by rapid compression, and the corresponding dashed lines to the steady states to which these relax. The line and colour code thus follow Fig. 5. The currents shown also assume a prompt response of the neutral atmosphere velocity to the changes in the plasma angular velocity. Panel (a) shows the normalised plasma angular velocity profiles, as in Fig. 5 but now mapped to the ionosphere, (b) the height-integrated ionospheric Pedersen current intensity $\left(\mathrm{A} \mathrm{m}^{-1}\right)$, positive equatorward, (c) the corresponding total Pedersen current integrated in longitude around the pole (MA), also positive equatorward, and (d) the field-aligned current density just above the ionosphere $\left(\mu \mathrm{A} \mathrm{m}{ }^{-2}\right)$ positive upward.

(negative) current of $\sim 65 \mathrm{MA}$ at the boundary between the outer and middle magnetosphere.

The field-aligned current density just above the ionosphere is related directly to the co-latitude gradient of the total current (Eq. 4), and is shown in Fig. 6d. In the initial state (black line) the field-aligned current has small nearly constant negative (downward) values throughout the region of open field lines where the total Pedersen current increases essentially as the square of the distance from the axis. It then reverses to form a positive (upward) spike at the open-closed field line boundary where the total current falls, returns to small nearly constant negative values in the outer magnetosphere where the total current again increases, and then becomes strongly positive once more in the middle magnetosphere where the total current declines to zero. The peak upward currents at both the open-closed field line boundary and near the middle magnetosphere boundary with the outer magnetosphere are $\sim 0.3 \mu \mathrm{A} \mathrm{m}^{-2}$. The same basic four-region pattern occurs for all the steady-state systems in this model (e.g. the dashed lines in Fig. 6d), as in the previous models presented by Cowley et al. (2005). As the system becomes more compressed, however, the steady-state field-aligned current densities increase at the open-closed field line boundary due to the increasing angular velocity shear occurring across the boundary, and are reduced in the middle magnetosphere. The field-aligned current profiles for the transient states, however, are very different. For the compression to $65 R_{J}$ (blue solid line), the current density at the open-closed boundary is again enhanced due to the increased angular velocities on closed field lines, but then reverses only to very small negative values in the outer part of the outer magnetosphere, before becoming positive again throughout the remainder of the outer magnetosphere and in the middle magnetosphere. However, the positive values are weak throughout, $\sim 0.1 \mu \mathrm{A} \mathrm{m}^{-2}$ or less, with a discontinuity and a reduction in magnitude on passing across the boundary from the outer to the middle magnetosphere. For the compression to $45 R_{J}$ (red solid line), the current density at the open-closed boundary is enhanced even further due to the super-corotation on closed field lines, while the sense of the currents in the outer and middle magnetospheres are completely reversed compared with the initial steady state, while being of comparable or 


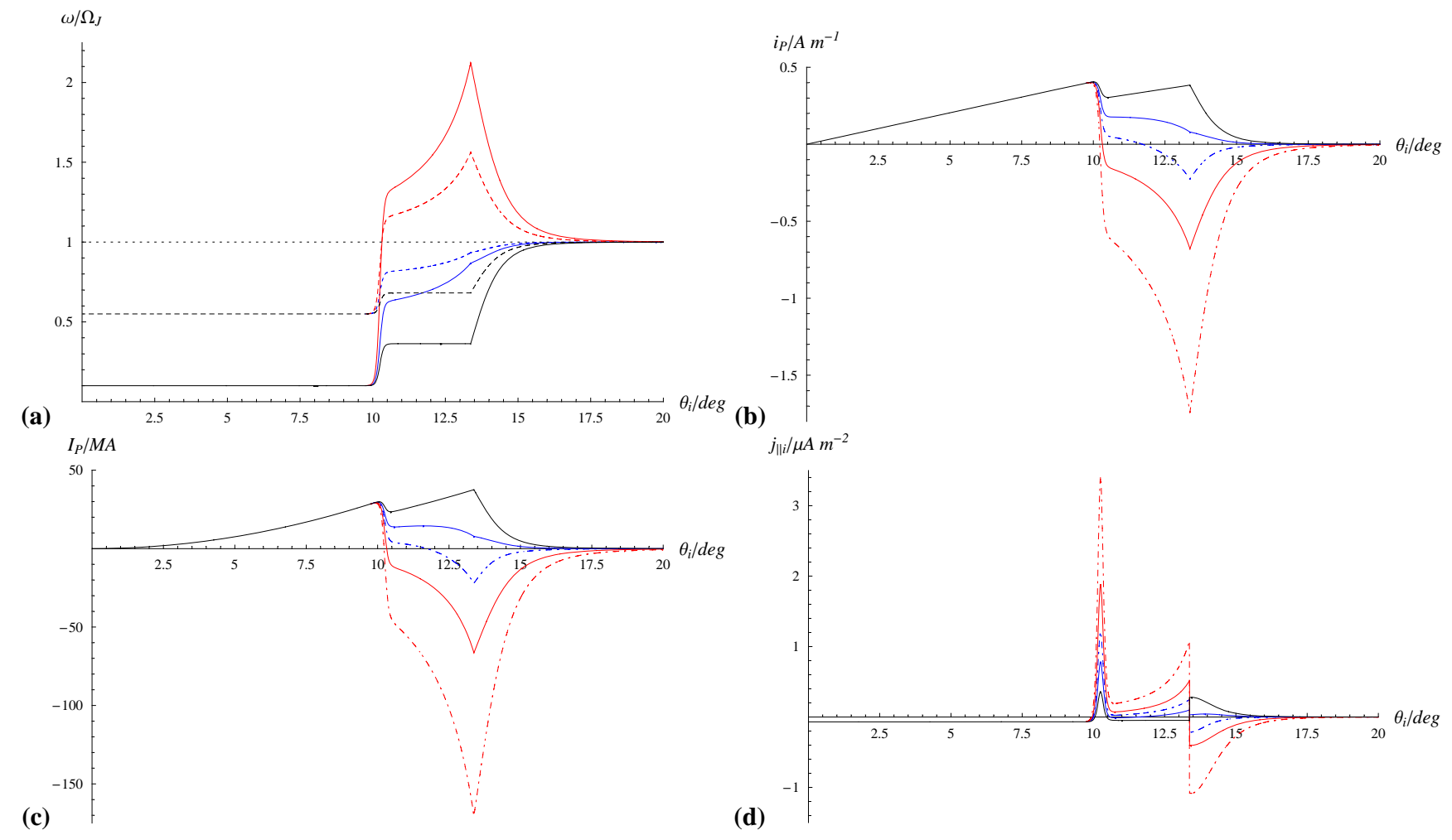

Fig. 7. Angular velocity profiles and ionospheric currents plotted versus ionospheric co-latitude for the case of compression of the magnetosphere similar to Fig. 6, but where we now compare profiles for the transient states assuming prompt responses of the neutral atmospheric flow on the compression time scale, and those for an unresponsive atmosphere. Panel (a) shows the angular velocities, where the solid lines show the plasma angular velocities in the initial state with a magnetopause at $85 R_{J}$ (black), and in the transient states formed by rapid compression inward to 65 (blue) and $45 R_{J}$ (red), as in Fig. 6 . The black, blue, and red dotted lines then show the neutral atmosphere angular velocities given by Eq. (5) in each case with $k=0.5$, the blue and red dotted lines then being the transient neutral profiles assumed in the prompt response results shown in Fig. 6. For an unresponsive atmosphere, however, the currents in the transient case are calculated using the black dotted line from the initial steady state throughout. In subsequent panels the transient cases using the unresponsive atmosphere assumption are shown by the dot-dashed lines, while those for the prompt atmospheric response are shown by the solid lines (as in Fig. 6), blue corresponding to the compression to $65 R_{J}$, and red to $45 R_{J}$, as previously. The black solid lines show results for the initial steady state (again as in Fig. 6). Using this format, panel (b) shows the height-integrated ionospheric Pedersen current intensity (A $\mathrm{m}^{-1}$ ), positive equatorward, (c) shows the corresponding total Pedersen current (MA), also positive equatorward, while (d) shows the ionospheric field-aligned current density $\left(\mu \mathrm{A} \mathrm{m}^{-2}\right)$ positive upward.

larger magnitude. In the transient state, upward-directed currents thus prevail across the whole outer magnetosphere region, with peaks at both the open-closed field line boundary $\left(\sim 1.7 \mu \mathrm{A} \mathrm{m}^{-2}\right)$ and at the interface with the middle magnetosphere $\left(\sim 0.5 \mu \mathrm{A} \mathrm{m}^{-2}\right)$. The field-aligned current is then directed downward throughout the middle magnetosphere region, as found previously by Cowley and Bunce (2003a, b), peaking in magnitude at $\sim 0.4 \mu \mathrm{A} \mathrm{m}^{-2}$ at the interface with the outer magnetosphere.

3.2.2 Results for an unresponsive atmosphere on the compression time scale

We now briefly compare these results with those obtained on the assumption that the neutral atmospheric flow does not re- spond on the compression time scale. These are shown in Fig. 7 in a format similar to Fig. 6, but also containing some important differences. The solid lines in Fig. 7a again show the initial steady-state and transient angular velocity profiles, exactly as in Fig. 6a. However, the coloured dotted lines now show the corresponding neutral atmospheric angular velocity profiles assumed in the prompt response results discussed in Sect. 3.2.1 above (Eq. 5 with $k=0.5$ in each case), such that the currents in that case are determined by the difference between the solid and dotted lines of corresponding colours. Now, however, if the neutral atmosphere does not respond on the compression time scale, the currents are instead determined from the difference between the solid lines and the black dotted line in each case, the latter corresponding to the atmospheric angular velocity in the initial steady state. It is 
thus readily seen that excitation of plasma super-corotation with respect to the neutral atmosphere, and consequent reversal in the sense of the current system, will occur for more moderate compressions in this case, as previously indicated by Gong and Hill (2005). The corresponding Pedersen current intensity profiles are shown in Fig. 7b, where the solid lines show the currents for the case of the prompt atmospheric response just as in Fig. 6b, while the dot-dashed lines show those for the unresponsive atmosphere case. Compression to $65 R_{J}$ is now sufficient to induce super-corotation with respect to the neutral atmosphere and reversal of the Pedersen current to poleward throughout the middle magnetosphere and in the inner part of the outer magnetosphere. Compression to $45 R_{J}$ produces super-corotation with respect to both the planet and neutral atmosphere throughout the closed field line region as before, but now produces current intensities more than a factor of two larger than in the latter case. We note that the steady states to which these systems ultimately relax are the same as those shown in Fig. 6b, and are not repeated in the figure. In Figs. $7 \mathrm{c}$ and d we similarly show the corresponding profiles of the total Pedersen current, and the ionospheric field-aligned current density determined from its gradient. The peak total current on closed field lines is now directed poleward in both cases, peaking at $\sim 20 \mathrm{MA}$ for the case of compression to $65 R_{J}$, and at $\sim 170$ MA for compression to $45 R_{J}$. The corresponding field-aligned current system on closed field lines is now fully reversed in sense even for compression to $65 R_{J}$, while the reversed currents for compression to $45 R_{J}$ are more than a factor of two larger than for the prompt atmosphere case.

\subsection{Power inputs to the magnetosphere and atmosphere}

We now consider the consequences of these current systems, and begin by examining the power input into the magnetosphere, and into atmospheric heating. Figure 8a shows colatitude profiles of the power per unit area transferred from planetary rotation into the magnetosphere given by Eq. (6), corresponding to the current systems for the prompt atmospheric response shown in Fig. 6b, and using the same line and colour format. It can be seen that in all the steadystate models the power values are of order a few tenths of a $\mathrm{W} \mathrm{m}^{-2}$, or less. Integrating over the closed field region yields total power inputs into equatorial plasma rotation (per hemisphere) of 189,179 , and $101 \mathrm{TW}$ for the models with magnetopauses at 85,65 , and $45 R_{J}$ (the black solid line and the blue and red dashed lines), respectively. Closed field line integrated values per hemisphere for these steady state models are shown by the dashed line in Fig. 8b, plotted over the range of magnetopause distances from 40 to $85 R_{J}$. The relative invariance of these magnetospheric powers results from the fact that as the plasma angular velocity increases towards rigid corotation with increased compression, so the Pedersen current intensity falls, such that these effects tend to cancel in Eq. (6) over the relevant range. For comparison, the power input to the magnetosphere integrated over the open field line region, which is also essentially unchanging due to the assumed unchanging nature of the flow, is $\sim 23.5 \mathrm{TW}$. The results for the transient states in Fig. 8a show that values for the modestly sub-corotating system produced by a compression to $65 R_{J}$ (blue solid line) are similar to the steady states, the integrated power over the closed field region in this case being 118 TW, as indicated by the solid line in Fig. 8b. However, for the case of compression to $45 R_{J}$ it can be seen in Fig. 8a that the power is large and negative throughout the closed field region, meaning that in this case the direction of power transfer is reversed, from magnetosphere to planet, through the mechanism of ion-neutral collisions in the Pedersen layer. The magnitude of the power peaks in this case at $\sim 4 \mathrm{~W} \mathrm{~m}^{-2}$ at the boundary between the outer and middle magnetosphere, while the total power integrated over the closed field region in the model is $-878 \mathrm{TW}$. The solid line in Fig. $8 \mathrm{~b}$ shows that the closed field integrated magnetospheric power in the transient states declines to zero at a magnetopause radius of $\sim 59 R_{J}$, at which point the plasma nearrigidly corotates throughout the closed field region (modestly super-corotating in the inner part and modestly subcorotating in the outer part). It then decreases rapidly to large negative values as the plasma is induced to strongly supercorotate throughout the closed field region. The dot-dashed line in this panel shows corresponding results for the transient states assuming an unresponsive atmospheric flow on the compression time scale. The closed field magnetospheric power passes through zero for a more modest compression to $\sim 68 R_{J}$ in this case, and reaches much larger negative values for large compressions than for the case of the prompt atmospheric response.

Figure $8 \mathrm{c}$ similarly shows co-latitude profiles of the power dissipated to atmospheric heating given by Eq. (7), corresponding to the currents shown in Fig. 6b. For this prompt atmospheric response case this is a positive definite quantity which goes just to zero when the plasma exactly rigidly corotates with the planet. Typical values are several tenths of a $\mathrm{W} \mathrm{m}^{-2}$. However, for the steady-state cases the values in the region of closed field lines fall continuously with increasing compression of the system, as the angular velocities successively approach rigid corotation. Integrated over the closed field region, the steady state atmospheric powers are 226, 78, and $14 \mathrm{TW}$ for magnetopause distances of 85 , 65 , and $45 R_{J}$, respectively. This variation is shown by the dashed line in Fig. 8d, which has the same format as Fig. 8b. For comparison, the essentially unchanging power integrated over open field lines is $\sim 195$ TW. For the transient states in Fig. $8 \mathrm{c}$, the integrated closed field value is similarly modest when the magnetopause is compressed in to $65 R_{J}$, equal to $43 \mathrm{TW}$, due to the near-corotational nature of the plasma in this case. However, for the transient state corresponding to compression to $45 R_{J}$, the atmospheric heating peaks at over $2 \mathrm{~W} \mathrm{~m}^{-2}$ at the boundary between the outer and middle magnetosphere, while the closed field integrated power 

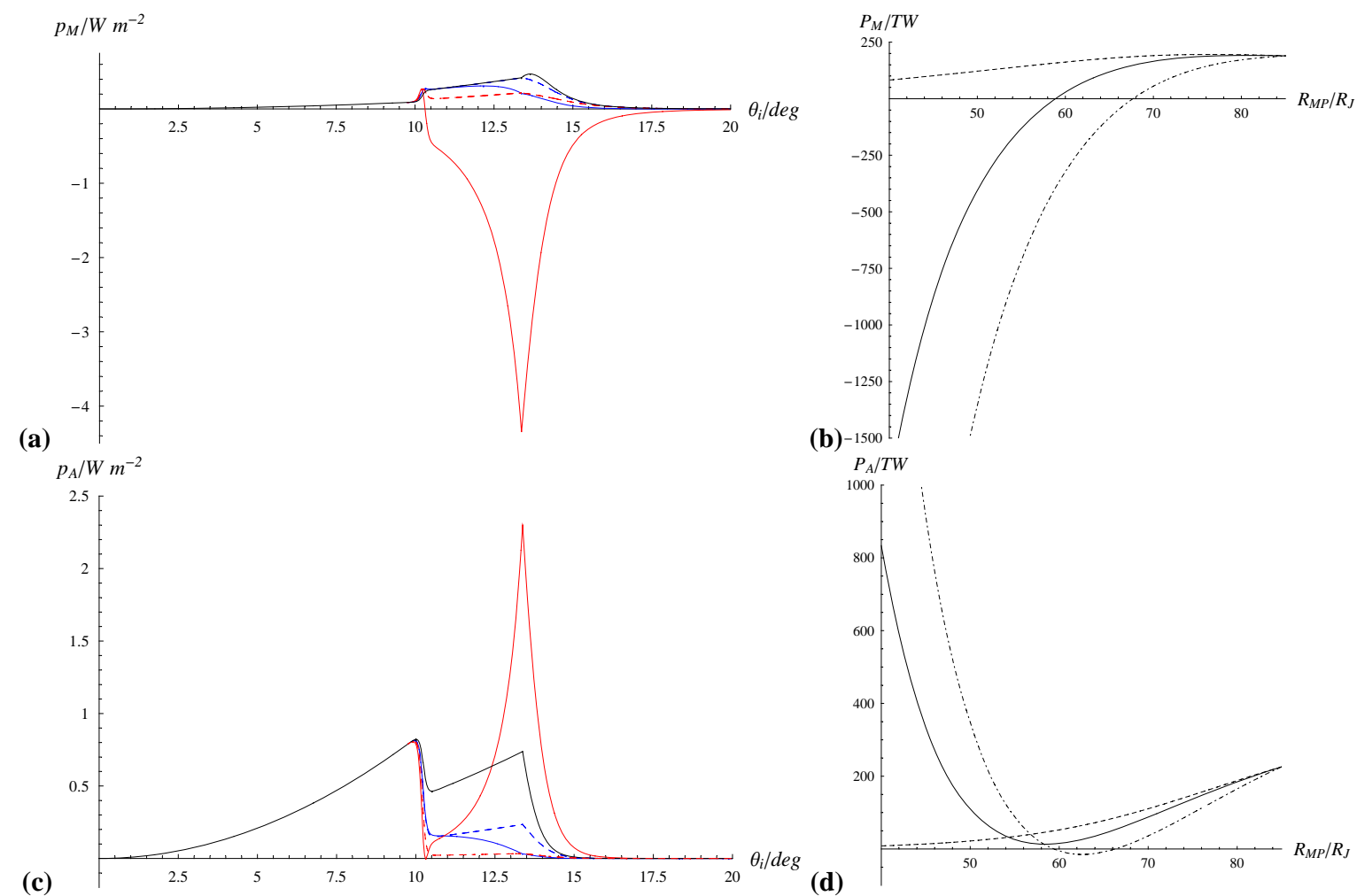

Fig. 8. (a) Co-latitude profile of power per unit area transferred from the planet to the magnetosphere given by Eq. (6), for the case of magnetospheric compression from an initial radius of $85 R_{J}$ to 65 and $45 R_{J}$, assuming a prompt atmospheric response to the changed plasma angular velocities. The line and colour codes follow those in Figs. 5 and 6. (b) Total power input to the magnetosphere (per hemisphere) integrated over the region of closed field lines, plotted versus the magnetopause radius $R_{M P}$ over the range from 40 to $85 R_{J}$. The solid line shows results for the transient states formed by compression from an initial radius of $85 R_{J}$ assuming a prompt response of the neutral atmospheric flow (thus corresponding to integrals under the solid lines in panel (a) at the appropriate $R_{M P}$ values), while the dashed line shows results for the steady-state models (thus corresponding to integrals under the dashed lines in panel a). The dot-dashed line shows corresponding results for the transient states assuming an unresponsive atmospheric flow on the compression time scale. The corresponding essentially constant concurrent power integrated over the region of open field lines is $\sim 23.5$ TW. (c) As for panel (a) but now for the power per unit area dissipated as atmospheric heating given by Eq. (7). (d) As for panel (b) but for the power per hemisphere dissipated to atmospheric heating on closed field lines (the values thus correspond to integrals under the lines in panel c). The corresponding essentially constant concurrent power integrated over the region of open field lines is $\sim 195 \mathrm{TW}$.

is increased to $334 \mathrm{TW}$. Overall results for the power per hemisphere dissipated to atmospheric heating in the closed field region in the transient states, shown by the solid line in Fig. 8d, shows a minimum for compression to $\sim 58 R_{J}$ where the plasma is near-rigidly corotating, and then a major increase with decreasing magnetopause radius as supercorotation is induced. Similar behaviour also occurs for the case of unresponsive atmospheric flow, shown by the dotdashed line in Fig. 8d. Like the magnetospheric power, however, this reduces to small values for more modest compressions of the system, and then increases to significantly larger values as the compression of the system increases, compared with the prompt atmospheric case.
3.4 Auroral electron acceleration and precipitating energy flux

\subsubsection{Auroral electron acceleration parameters for prompt atmospheric flow response case}

We now turn to the auroral consequences of the field-aligned currents, and begin in Fig. 9 by showing the auroral acceleration parameters corresponding to the current systems in Fig. 6 (using the same line and colour codes), which result from system compression from $85 R_{J}$ to 65 and to $45 R_{J}$, with prompt response of the atmospheric flow. Figure 9a shows the field-aligned current density as in Fig. 6d, but now displayed on an expanded co-latitude scale between $8^{\circ}$ and $18^{\circ}$, thus focussing on the region spanning the open-closed field line boundary and the high-latitude region of closed 

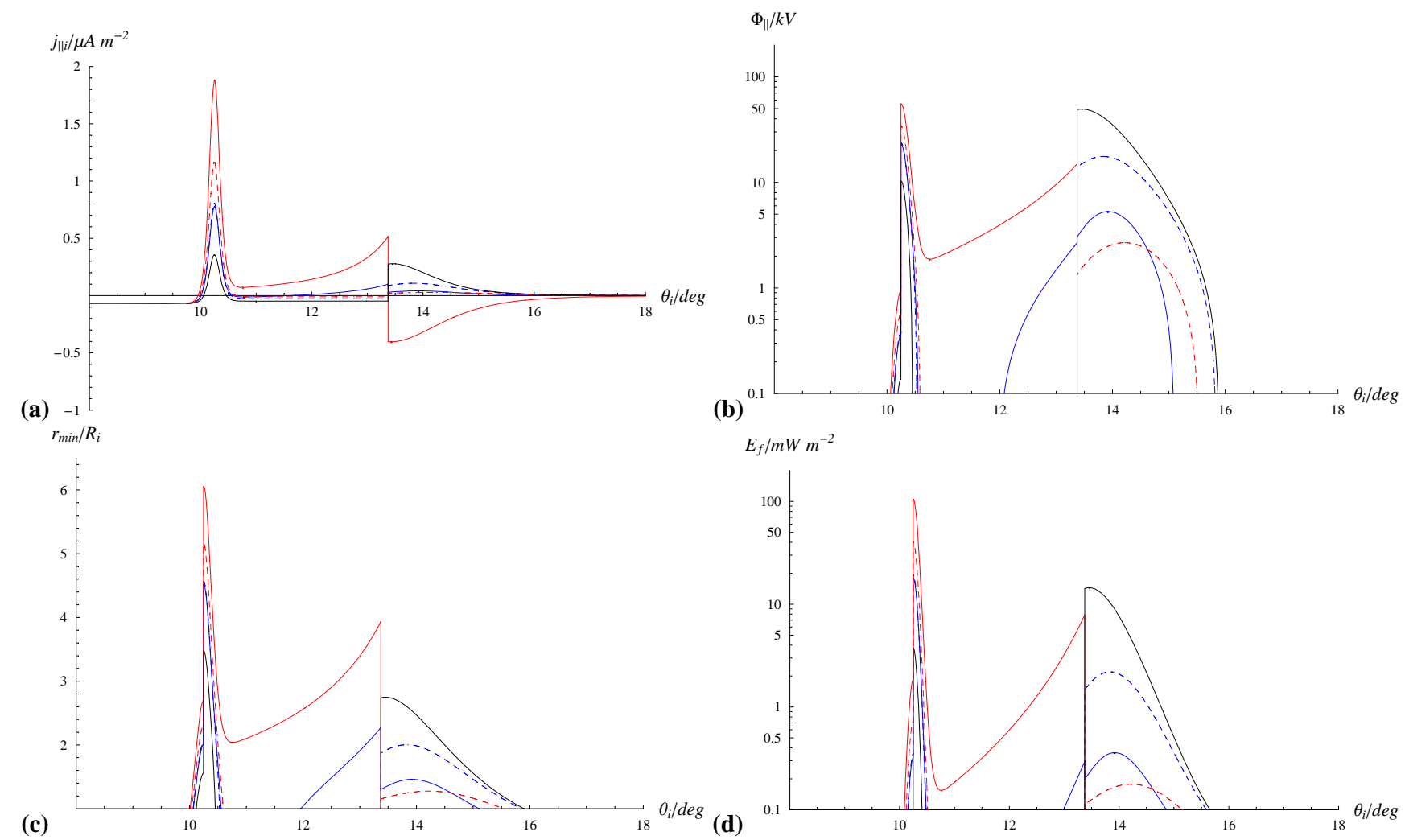

(c)

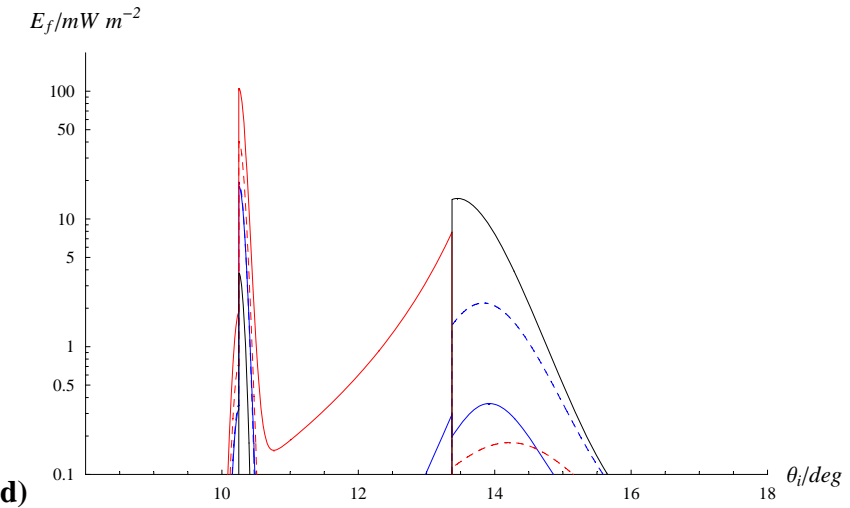

Fig. 9. Plots showing auroral acceleration parameters in regions of upward-directed field-aligned current for the case of magnetospheric compression from an initial value of $85 R_{J}$ to 65 and $45 R_{J}$, assuming a prompt atmospheric response to the changed plasma angular velocities. The line and colour codes follow those in Figs. 5 and 6 . The parameters are plotted versus co-latitude over the range $8^{\circ}-18^{\circ}$, spanning the open-closed field line boundary and the closed field region of interest. The panels show (a) the field-aligned current density $\left(\mu \mathrm{A} \mathrm{m}^{-2}\right)$ as in Fig. 6d, (b) the accelerating voltage $(\mathrm{kV})$ in upward-directed field-aligned current regions from Eq. (23) employing a log scale, (c) the minimum radial distance of the acceleration region along the field lines in units of the polar ionospheric radial distance $R_{i}$ $\left(\sim 0.94 R_{J}\right)$ from Eq. (24), and (d) the precipitating electron energy flux $\left(\mathrm{mW} \mathrm{m}^{-2}\right)$ from Eq. (25), again on a log scale.

field lines. To consider the requirements for acceleration, we compare the upward-directed (positive) current densities in this plot with the limiting currents that can be carried by the unaccelerated magnetospheric electrons in the three regions considered, namely open field lines, the outer magnetosphere, and the middle magnetosphere, as shown in Table 1 . These are $\sim 0.1 \mu \mathrm{A} \mathrm{m}^{-2}$ for the magnetosheath (cusp) source, taken here to apply to the open field line region immediately poleward of the open-closed field line boundary (and hence to the upward current at the boundary poleward of the peak), and $\sim 0.01 \mu \mathrm{A} \mathrm{m}^{-2}$ in both the outer and middle magnetosphere regions. It can be seen that the upward current in Fig. 9a generally considerably exceeds these limiting values, such that downward acceleration of magnetospheric electrons is required to carry them. The accelerating voltages obtained from Eq. (23) combined with the source parameters in Table 1, are plotted versus co-latitude in Fig. 9b. Similarly, the minimum height of the acceleration region obtained from Eq. (24) is shown in Fig. 9c in units of the ionospheric radius
$R_{i}\left(\sim 0.94 R_{J}\right)$, while the precipitating energy flux obtained from Eq. (25) is shown in Fig. 9d.

If we consider the steady-state solutions first, it can be seen that there are always two separated regions of upwarddirected current and accelerated electron precipitation, one corresponding to the open-closed field line boundary, the other to the middle magnetosphere, and that with increasing compression of the system the former grows in intensity while the latter diminishes. In all the solutions the plasma angular velocity increases from $10 \%$ of rigid corotation on open field lines to full rigid corotation at lower latitudes, and as the system becomes more compressed more of this flow shear falls across the open-closed boundary and less across the middle magnetosphere. At the open-closed boundary the accelerating voltages are typically a few hundred volts where the current is taken to be carried by magnetosheath electrons poleward of the boundary, increasing to a few tens of kilovolts where the current is taken to be carried by the outer magnetospheric electrons equatorward 
of the boundary. These differences reflect the lower density and higher temperature of the outer magnetosphere electrons compared with the magnetosheath. The resulting energy fluxes are typically a few tenths of a $\mathrm{mW} \mathrm{m}^{-2}$ on the open field line side of the boundary, sufficient to produce an aurora of only a few $\mathrm{kR}$, while increasing to a few to a few tens of $\mathrm{mW} \mathrm{m}^{-2}$ on the closed field line side of the boundary, sufficient to produce bright auroras of a few tens to a few hundreds of kR. Integrating over the whole open-closed field line boundary region, defined here to be the region of total width $0.75^{\circ}$ centred on the open-closed field boundary which encompasses the boundary current in the model (thus lying between $\sim 9.9^{\circ}$ and $\sim 10.6^{\circ}$ co-latitude), the precipitating electron power in the model increases from $26 \mathrm{GW}$ in the initial steady-state when the magnetopause lies at $85 R_{J}$, to $143 \mathrm{GW}$ when it is compressed to $65 R_{J}$, and to $306 \mathrm{GW}$ when it is compressed to $45 R_{J}$. The corresponding UV auroral output is $\sim 10 \%$ of these values. The total precipitating electron power into this region if the populations remained unaccelerated at the energy fluxes given by Eq. (22) would amount to only $\sim 0.45 \mathrm{GW}$. We also note from Fig. $9 \mathrm{c}$ that to provide the necessary electron flux for the boundary current, the acceleration region must lie high on the field lines on the closed field side of the boundary, above $\sim 3-5 R_{i}$, depending on the state of compression. The acceleration region can lie much closer to the planet on the open field side of the boundary where the current is taken to be carried by the denser cusp electrons.

We should perhaps note at this point that the detailed values of the auroral parameters computed at the open-closed field line boundary, as above, do depend on the model assumption concerning the latitudinal spatial scale on which the changes at the boundary take place. The change in angular velocity at the boundary determines the total field-aligned current flowing for given ionospheric conductivity through Eqs. (3) and (4), while the current density, and hence the accelerating field-aligned voltage and precipitating energy flux, is also determined by the assumed width of the fieldaligned current layer parameterised by $\Delta \theta_{i} M P$ in Eq. (19). The value adopted here corresponds to a north-south spatial scale of $\sim 440 \mathrm{~km}$ as indicated in Sect. 2.5, which is hopefully reasonable. However, for other choices, the current density and accelerating voltage (Eq. 23) will vary inversely with the chosen width, while the precipitating electron energy flux and UV luminosity will vary inversely as the square of the width (Eq. 25). Integrating over the whole boundary region, therefore, the total precipitating electron power will vary inversely with the assumed layer width. This level of uncertainty, which is not shared by the other integrated powers computed in this paper, should be borne in mind when considering the results for this region which we present.

For the middle magnetosphere regions in the steady-state solutions, it can be seen in Fig. $9 \mathrm{~b}$ that the peak accelerating voltages fall with the field-aligned current density from $\sim 50 \mathrm{kV}$ in the initial expanded state, to $\sim 20$ and $\sim 3 \mathrm{kV}$ as the system is compressed inwards to 65 and $45 R_{J}$, respectively. The minimum radial distances of the acceleration region in Fig. 9c fall correspondingly, but are never greater than $\sim 3 R_{i}$. With regard to the precipitating electron power shown in Fig. 9d, it can be seen that the peak powers are sufficient to produce an aurora of $\sim 100 \mathrm{kR}$ in intensity in the initial state, reducing to $\sim 20$ and $\sim 2 \mathrm{kR}$ as the compression increases. The total powers integrated over the middle magnetosphere region from the boundary with the outer magnetosphere to $18^{\circ}$ co-latitude, fall from $1.28 \mathrm{TW}$ in the initial state, to 327 and $60 \mathrm{GW}$ for compressions to 65 and $45 R_{J}$, respectively. The unaccelerated power input in the middle magnetosphere to $18^{\circ}$ at the rate given by Eq. (22) is $42 \mathrm{GW}$. In essence, a magnetopause compression inwards to $45 R_{J}$ effectively suppresses the accelerated middle magnetosphere precipitation in the steady state.

Now examining the transient cases shown in Fig. 9, it is first seen that the response at the open-closed field line boundary is similar to that described above, due to the increased flow shear that occurs across the boundary in compressed conditions, whether transient or steady-state. However, the auroral acceleration on closed field lines equatorward of the boundary is distinctly different, reflecting the differing patterns of field-aligned current in Fig. 9a. When the system is compressed to $65 R_{J}$ (blue solid lines), downward electron acceleration is required in a region on both sides of the boundary between the outer and middle magnetospheres. The accelerating voltages in Fig. $9 \mathrm{~b}$ are typically a few $\mathrm{kV}$, peaking at $\sim 5 \mathrm{kV}$ in the middle magnetosphere. The voltages are generally smaller in the outer magnetosphere region despite the peak field-aligned currents being larger, due to the lower temperature of the source plasma in that region compared with the middle magnetosphere (Table 1). The peak electron energy fluxes shown in Fig. 9d are sufficient to produce auroras of only a few $\mathrm{kR}$, however, with total integrated powers of 76 and $12 \mathrm{GW}$ in the middle and outer magnetosphere regions, respectively. The latter value compares with a total unaccelerated precipitated power in the outer magnetosphere region of $1.2 \mathrm{GW}$, given by Eq. (22). For comparison, the total precipitating electron power in the open-closed boundary region, defined as above, is $\sim 137 \mathrm{GW}$ in this case. This transient state represents a transitional configuration between the two-zone accelerated precipitation pattern characteristic of steady-state conditions, and that associated with the excitation of super-corotation throughout the closed field region of the model such as occurs for a magnetopause compression to $45 R_{J}$ (red solid lines). In this case, downward electron acceleration is required throughout the outer magnetosphere region from the open-closed field line boundary as shown in Fig. 9b, the required voltages increasing from $\sim 2$ to $\sim 15 \mathrm{kV}$ across this region before switching off across the boundary with the middle magnetosphere where the fieldaligned current reverses to downward. The acceleration region must also be located relatively high on the outer magnetosphere field lines to provide the required electron flux 
(a)

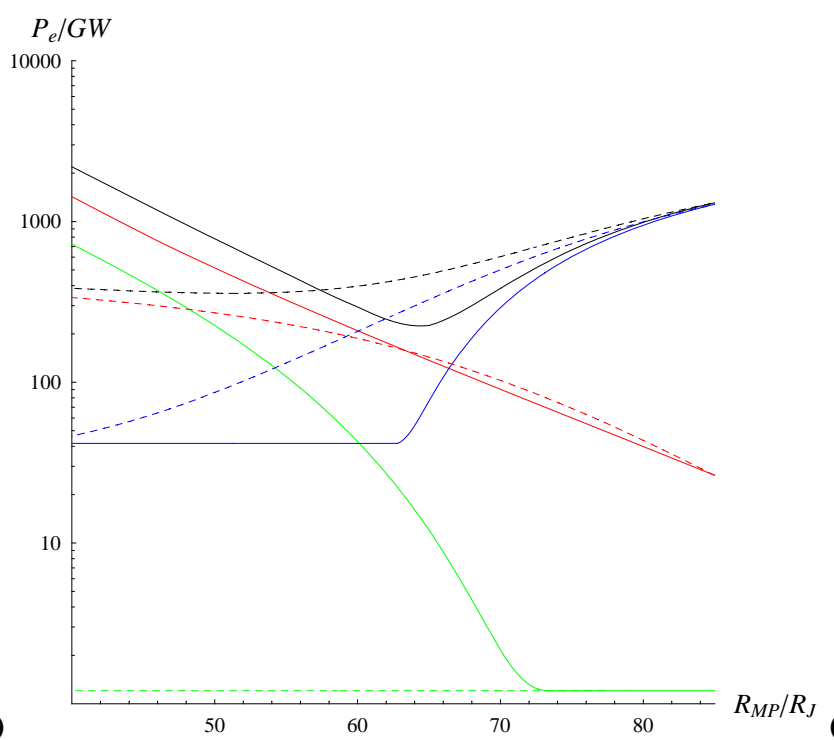

(b)

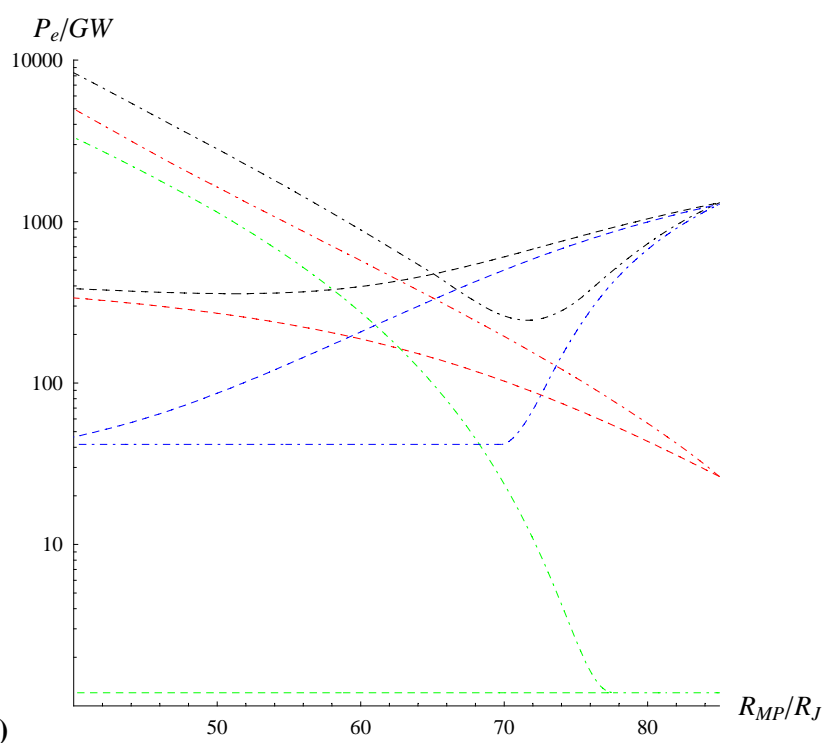

Fig. 10. (a) Overview of the variation of precipitating electron power per hemisphere (log scale) integrated over various regions of the model versus the magnetopause radius $R_{M P}$ in $R_{J}$, for the case of rapid system compression from a magnetopause radius of $85 R_{J}$, assuming a prompt response of the atmospheric flow. Red lines show the power input into the open-closed field line boundary region (defined as the region of latitudinal width $0.75^{\circ}$ centred on the open-closed field line boundary), green lines the outer magnetosphere region, blue lines the middle magnetosphere to $18^{\circ}$ co-latitude, while the black lines show the sum of these components. Solid lines refer to the transient states formed by rapid compression inward from the initial magnetopause radius at $85 R_{J}$ (the right-hand border of the plot), while dashed lines show the steady-state values to which these revert after $\sim 1-2$ days of steady compression. (b) As for panel (a) but now for the case of an unresponsive atmospheric flow on the compression time scale. Results for the transient cases are now plotted using dot-dashed lines.

at the ionosphere, beyond radial distances of $\sim 2-4 R_{i}$ according to Fig. 9c. The electron energy flux produced by the acceleration then peaks at $\sim 10 \mathrm{~mW} \mathrm{~m}^{-2}$ at the boundary between the outer and middle magnetosphere regions, sufficient to produce a $\sim 100 \mathrm{kR}$ aurora, while falling towards a minimum of $\sim 0.1 \mathrm{~mW} \mathrm{~m}^{-2}$ and $\sim 1 \mathrm{kR}$ near the open-closed field line boundary. It can thus be seen that in effect a two-ring auroral distribution will still result under these conditions, with the lower-latitude "ring" peaking at the boundary between the outer and middle magnetosphere, at essentially the same latitude that the middle magnetosphere current peaks in the steady-state solutions. However, the bright auroral region now extends poleward from that boundary toward the open field region, rather than towards lower latitudes as in the steady state cases. The total precipitating electron power in the outer magnetosphere region is found to be $418 \mathrm{GW}$ under this condition, compared with $841 \mathrm{GW}$ into the open-closed field line boundary defined as above, and $42 \mathrm{GW}$ for the unaccelerated population precipitating into the middle magnetosphere region (to $18^{\circ}$ co-latitude).

\subsubsection{Overview of precipitating electron powers}

Overall results for the integrated auroral powers per hemisphere for the case of magnetospheric compression with a prompt atmospheric response are shown in Fig. 10a, plot- ted on a log scale versus the radial distance of the magnetopause. Results for the various regions of the model are colour-coded, red for the open-closed field line boundary region (defined as above), green for the outer magnetosphere, blue for the middle magnetosphere, and black for total values. We recall that the UV output powers are $\sim 10 \%$ of these values. Results pertaining to steady-state solutions are shown by dashed lines, while those for the transient states produced by a compression inward from $85 R_{J}$ are shown by the solid lines. If we look first at the steady-state results shown by the dashed lines, it can be seen that the auroral power associated with the middle magnetosphere (blue dashed line) dominates the expanded system, but strongly declines with increasing compression, as in the examples shown above. Conversely, the power associated with the open-closed field line boundary (red dashed line) grows strongly with increasing compression, becoming dominant for magnetopause radii less than $\sim 59 R_{J}$. The power in the outer magnetosphere region (green dashed line) remains fixed at the unaccelerated limiting value throughout in this case, since the current in that region is always directed downward in the steady state. Adding these components together results in the total precipitated electron power shown by the black dashed line, which overall declines from $1.31 \mathrm{TW}$ for the expanded state at $85 R_{J}$, to $384 \mathrm{GW}$ for the compressed state at $40 R_{J}$. 
For the transient states it can be seen that the middle magnetosphere power (blue solid line) declines rapidly with increasing compression, reaching the unaccelerated limiting value of $43 \mathrm{GW}$ at a magnetopause position of $\sim 63 R_{J}$ (just inside the radius illustrated in the $65 R_{J}$ example above). On the other hand, the power input into the outer magnetosphere (green solid line) rises above its unaccelerated limiting value for magnetopause radii less than $\sim 73 R_{J}$, and increases rapidly with increasing compression, exceeding the unaccelerated value for the middle magnetosphere for magnetopause radii less than $\sim 60 R_{J}$, and reaching $723 \mathrm{GW}$ at $40 R_{J}$. By comparison, the precipitating electron power in the open-closed field line boundary region (red solid line) increases from modest values for the expanded system to become dominant for magnetopause radii less than $\sim 66 R_{J}$. Overall, the total precipitating electron power in the transient states declines from 1.31 TW for the initial expanded system at $85 R_{J}$, reaches a minimum value of $219 \mathrm{GW}$ at $\sim 64 R_{J}$, and increases again with further compression to $2.19 \mathrm{TW}$ at $40 R_{J}$.

A similar overview for the unresponsive atmosphere case is shown in Fig. 10b, the current systems for which were discussed in Sect. 3.2.2 and shown in Fig. 7. The format is the same as Fig. 10a, except that values for the transient states are shown by dot-dashed lines (as in Fig. 7), as opposed to the solid lines in Fig. 10a. Values for the steady states shown by the dashed lines are identical in the two plots. The same basic pattern of results can be discerned in this plot as in Fig. 10a, and for the same reasons. However, the excitation of accelerated electron input in the outer magnetosphere, and the related switching off of that in the middle magnetosphere, occurs for more modest compressions than in Fig. 10a, and the subsequent growth of the accelerated electron power for larger compressions is significantly greater. In this case the total electron power input in the transient state exceeds that in the initial state for compressions inside $\sim 57 R_{J}$, corresponding to solar wind dynamic pressures exceeding $\sim 0.12 \mathrm{nPa}$, and reaches $\sim 8.36 \mathrm{TW}$ for compressions to $40 R_{J}$.

\section{Magnetospheric expansion}

We now consider the effect of magnetospheric expansions caused by reductions in solar wind dynamic pressure, and for purposes of illustration consider the case of sudden expansions from an initially highly compressed state with a magnetopause at $45 R_{J}$, corresponding to a solar wind compression region, outward to 65 and to $85 R_{J}$. The order of discussion will follow that of the previous section.

\subsection{Plasma angular velocity profiles}

In Fig. 11 we show the plasma angular velocity profiles plotted versus radial distance in the equatorial plane, where the line and colour code follows that in previous sections.

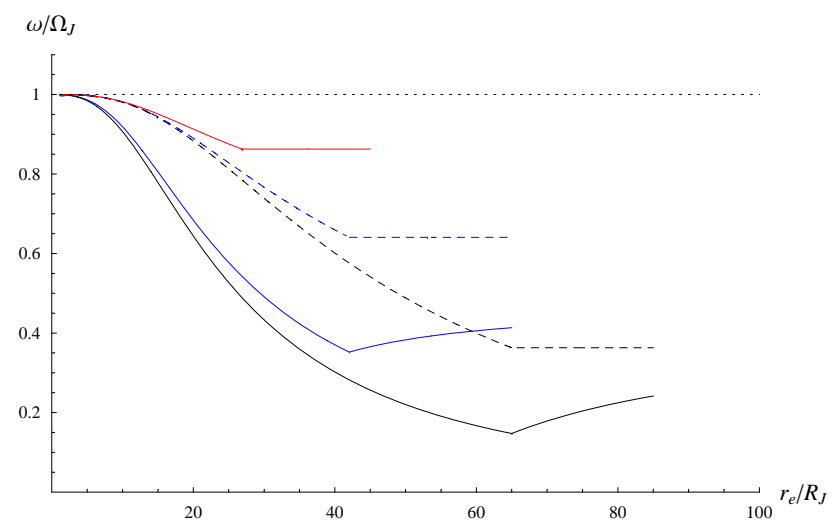

Fig. 11. Normalised plasma angular velocity profiles plotted versus equatorial radial distance for the case of an expansion of the magnetopause due to a sudden fall in solar wind dynamic pressure. The red solid line shows the initial steady state with a magnetopause at $45 R_{J}$. The blue and black solid lines then show the transient profiles following an outward expansion of the magnetopause to 65 and $85 R_{J}$, respectively. The blue and black dashed lines show the steady states to which these profiles relax after a $\sim 1-2$ day interval. The horizontal dotted line shows the condition of rigid plasma corotation.

Specifically, red, blue, and black lines correspond to magnetopause positions of 45,65 , and $85 R_{J}$, respectively. The red solid line thus shows the initial steady state profile corresponding to a magnetopause at $45 R_{J}$, in which the plasma is close to rigid corotation throughout, with $\left(\omega / \Omega_{J}\right)$ falling to $\sim 0.85$ at the outer boundary of the middle magnetosphere and in the outer magnetosphere. These steady states are of course the same for a given magnetopause radius as those shown in Sect. 3. The blue and black solid lines then show the angular velocities in the transient states produced by rapid magnetopause expansion from $45 R_{J}$ to 65 and $85 R_{J}$, respectively, while the blue and the black dashed lines show the corresponding steady states to which these relax. It can be seen that on sudden expansion, the plasma angular velocities drop to low values throughout much of the middle and outer magnetosphere, and then increase again somewhat as steady conditions are resumed. The decreases in angular velocity seen in the figure inside $\sim 20 R_{J}$ are somewhat exaggerated, however, since the magnitude of the model field employed for the middle magnetosphere becomes unrealistically small within this distance, so that the field line displacements are unrealistically large.

\subsection{Magnetosphere-ionosphere coupling current system}

\subsubsection{Results for the case of prompt atmospheric flow re-} sponse

The ionospheric currents corresponding to these angular velocity profiles are shown in Fig. 12, in the same format as Fig. 6. Figure 12a thus shows the plasma angular velocity 

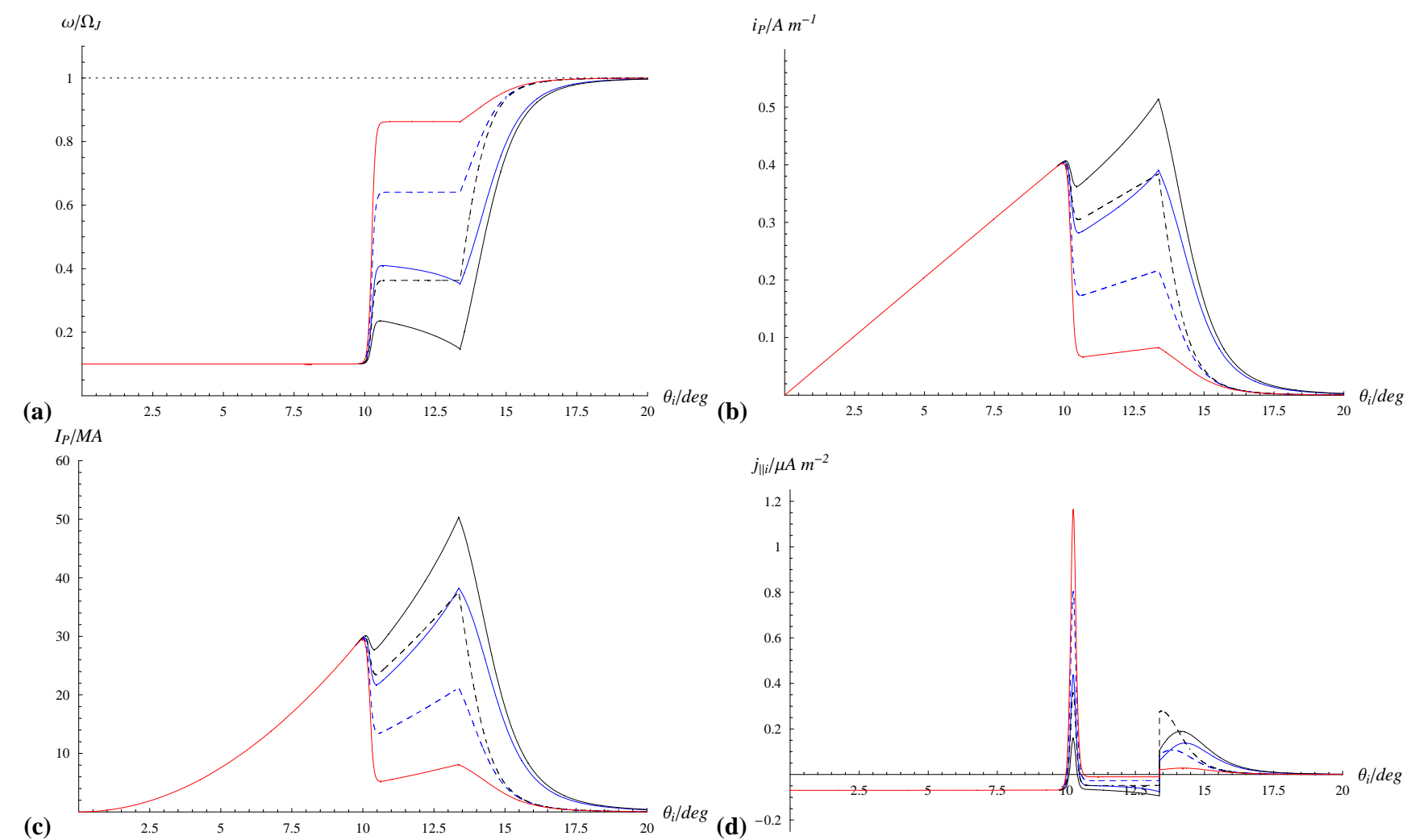

Fig. 12. Angular velocity and ionospheric current profiles plotted versus ionospheric co-latitude for the case of magnetospheric expansion, assuming a prompt response of the neutral atmosphere flow. The colour code follows Fig. 11, in which the red solid lines show the initial steady state with a magnetopause at $45 R_{J}$, the blue and black solid lines the transient states following magnetopause expansions to 65 and $85 R_{J}$, respectively, while the blue and black dashed lines show the steady states to which these relax. Panel (a) shows the normalised plasma angular velocity profiles, as in Fig. 11 but now mapped to the ionosphere, (b) shows the height-integrated ionospheric Pedersen current intensity, positive equatorward, (c) shows the corresponding total Pedersen current, also positive equatorward, while (d) shows the field-aligned current density just above the ionosphere positive upward.

profiles of Fig. 11 transformed into the ionosphere and plotted versus co-latitude. It can be seen that as the system expands, the flow shear at the open-closed field line boundary falls, while that across the middle magnetosphere increases. This applies both to the transient and steady states, but the effect is larger for the former than the latter. Figures $12 \mathrm{~b}$ and $\mathrm{c}$ then show the corresponding Pedersen current intensity and total Pedersen current profiles, respectively, where we are again assuming a prompt response of the atmospheric flow. In all these profiles the current falls at the boundary between open and closed field lines, rises again in the outer magnetosphere, and then falls to small values across the middle magnetosphere. However, due to the behaviour of the angular velocity, the fall at the open-closed field line boundary decreases with expansion of the system, and for the transient states compared with the steady states, and vice-versa for the fall across the middle magnetosphere. The consequences for the field-aligned current are shown in Fig. 12d. As then expected, this shows a consistently upward-directed current at the open-closed field line boundary, which reduces with in- creasing expansion of the system and for the transient states compared with the steady states. The currents in the outer and middle magnetosphere are then consistently downwardand upward-directed, respectively, and show an opposite behaviour in being enhanced for increasing expansion of the system, and for the transient states compared with the steady states.

\subsubsection{Results for an unresponsive atmosphere on the com- pression time scale}

Corresponding results for the case of an unresponsive atmospheric flow are shown in Fig. 13, in the same format as Fig. 7. In Fig. 13a the red solid line thus shows the initial plasma angular velocity profile, while the blue and black solid lines show the transient states formed by rapid expansion to 65 and to $85 R_{J}$, respectively, exactly as in Fig. 7a. The coloured dotted lines then show the corresponding atmospheric angular velocity profiles given by Eq. (5) with $k=0.5$, these being the atmospheric angular velocities assumed in 

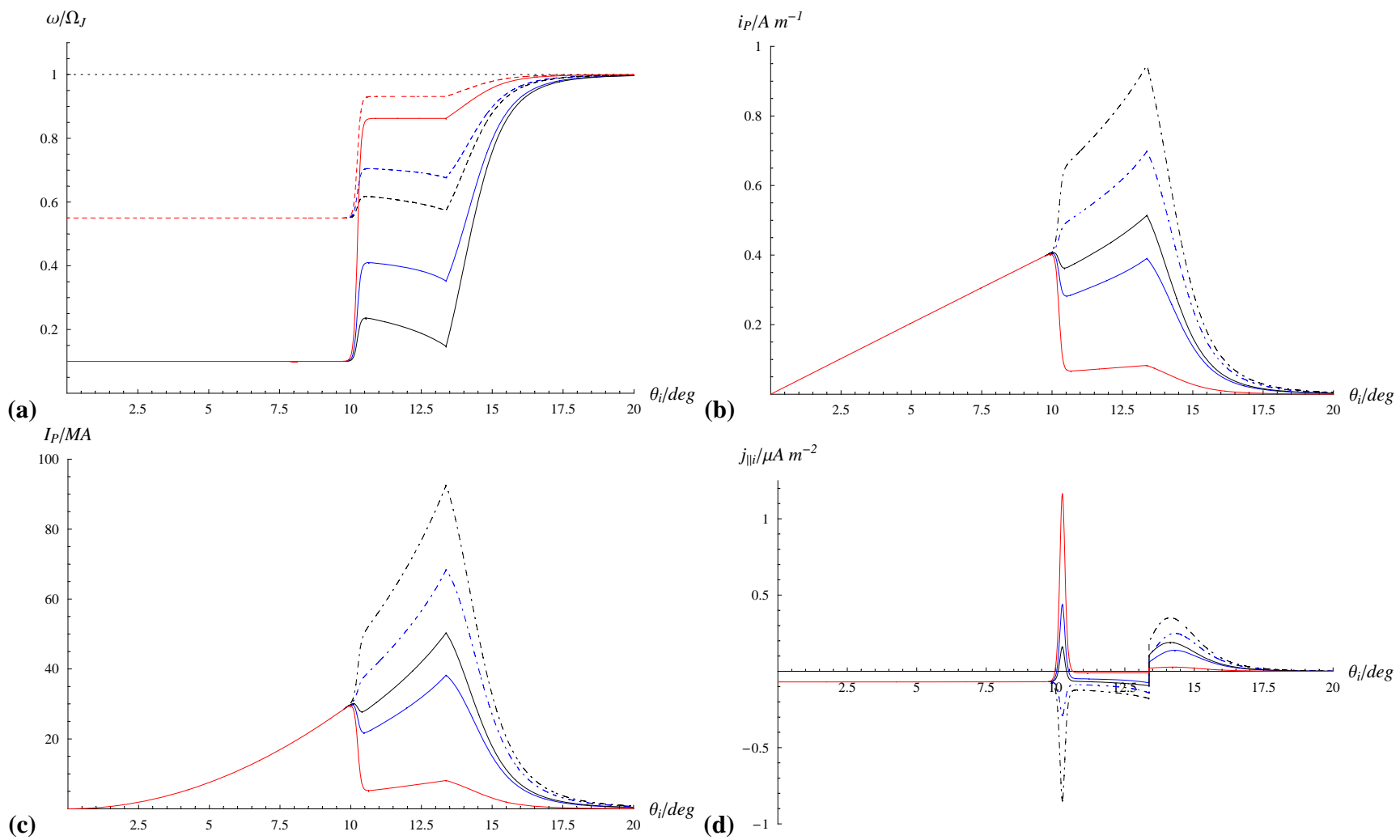

Fig. 13. Similar to Fig. 12, but now comparing transient case results for prompt and unresponsive atmosphere cases. Panel (a) shows the angular velocity profiles, where the solid lines show the plasma angular velocities in the initial state corresponding to a magnetopause at $45 R_{J}$ (red), and in the transient states formed by rapid expansion to 65 (blue) and $85 R_{J}$ (black), as in Fig. 12. The red, blue, and black dotted lines then show the neutral atmosphere angular velocities given by Eq. (5) in each case with $k=0.5$, the blue and black dotted lines being the transient neutral profiles assumed in the prompt response results shown in Fig. 12. In the unresponsive atmosphere case, however, the transient currents are calculated using the red dotted line from the initial steady state throughout. In subsequent panels the transient profiles for the unresponsive atmospheric case are shown by dot-dashed lines, while those for the prompt atmospheric response cases are shown for comparison by the solid lines, blue corresponding to expansion to $65 R_{J}$, and black to $85 R_{J}$. The red solid lines show results for the initial steady state. With this format, panel (b) shows the ionospheric Pedersen current intensity, positive equatorward, (c) shows the corresponding total Pedersen current, also positive equatorward, while (d) shows the ionospheric field-aligned current density, positive upward.

the prompt response case discussed in Sect. 4.2.1 above. The currents in the latter case are thus determined by the difference between the solid and dotted lines of the same colour, while for the unresponsive atmosphere case considered here they are determined by the difference between the solid lines and the red dotted line in each case. It can thus be seen that this will significantly increase the magnitude of the Pedersen currents on closed field lines. This is shown in Figs. 13b and c, where the solid lines show the Pedersen current intensity and total Pedersen current for the initial steady state (red) and for the transient expanded states assuming a prompt atmospheric response (blue and black) as in Fig. 12, while the blue and black dot-dashed lines show the modified transient profiles assuming an unresponsive atmosphere. Comparison shows that the Pedersen currents are increased roughly by a factor of two throughout the region of closed field lines in the latter cases. As a consequence, the Pedersen current intensity now increases sharply at the boundary between open and closed field lines, unlike all previous cases discussed in this paper, before increasing further across the outer magnetosphere and then falling rapidly to zero across the middle magnetosphere. The consequences for the field-aligned current profiles shown in Fig. 13d is that in these cases the current at the open-closed field line boundary is reversed to downward, such that the current is downward-directed throughout the region from the pole to the boundary between the outer and middle magnetosphere. Strong upward currents then flow throughout the middle magnetosphere. 
(a)

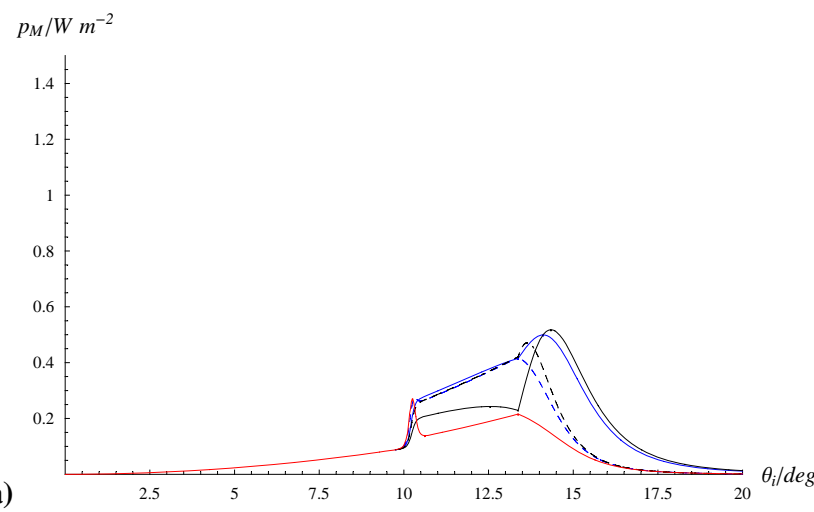

$p_{A} / W m^{-2}$

(c)

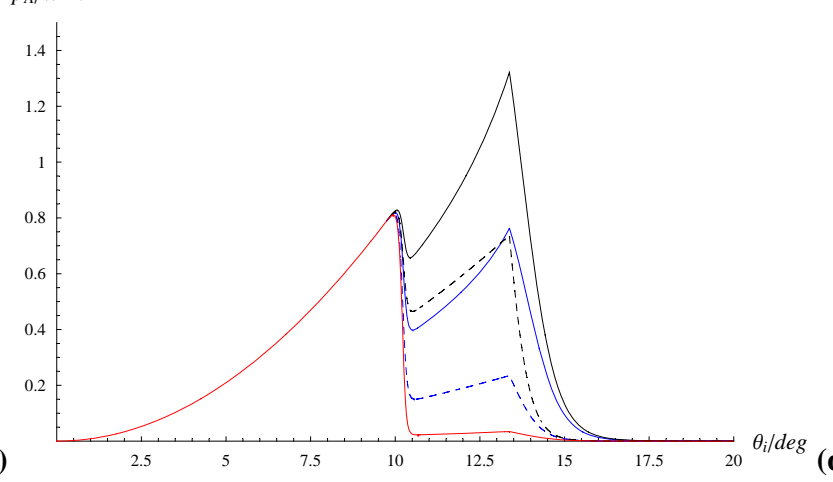

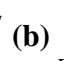

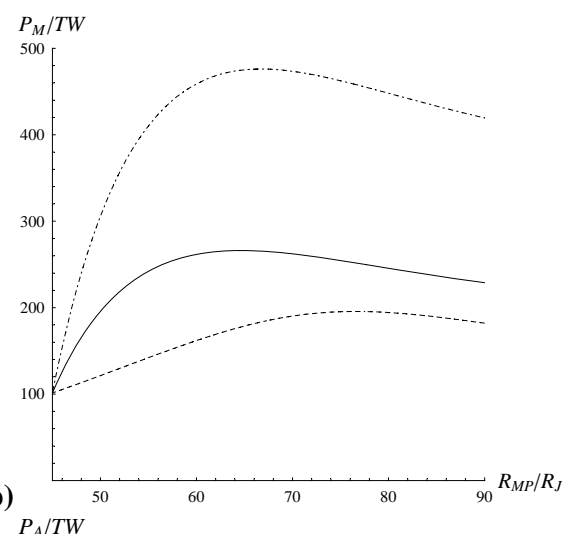

(d)

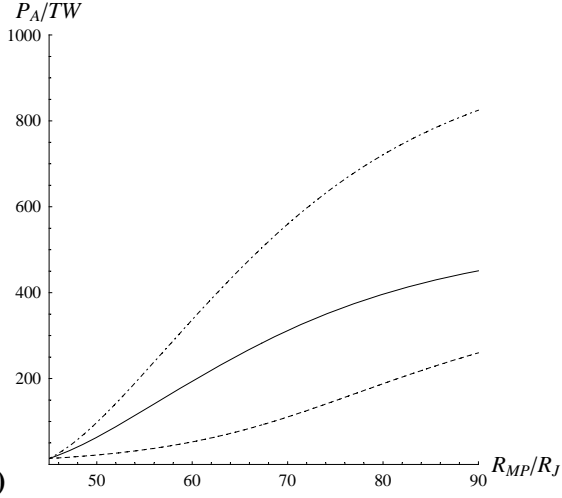

Fig. 14. (a) Co-latitude profile of power per unit area transferred to the magnetosphere for the case of magnetospheric expansion from an initial radius of $45 R_{J}$ to 65 and $85 R_{J}$, assuming a prompt atmospheric response to the changed plasma angular velocities. The line and colour codes follow those in Figs. 11 and 12. (b) Total power per hemisphere input to the magnetosphere (per hemisphere) integrated over the region of closed field lines, plotted versus the magnetopause radius $R_{M P}$ over the range from 45 to $90 R_{J}$. The solid line shows results for the transient states formed by expansion from an initial radius of $45 R_{J}$ assuming a prompt response of the neutral atmospheric flow (thus corresponding to integrals under the solid lines in panel (a) at the appropriate $R_{M P}$ values), while the dashed line shows results for the steady-state models (thus corresponding to integrals under the dashed lines in panel a). The dot-dashed line shows corresponding results for the transient states assuming an unresponsive atmospheric flow on the compression time scale. (c) As for panel (a) but now for the power per unit area dissipated as atmospheric heating. (d) As for panel (b) but now for the power dissipated per hemisphere to atmospheric heating in the closed field region (thus corresponding to integrals under the curves in panel c).

\subsection{Power inputs to the magnetosphere and atmosphere}

Figure 14 shows results for the power input to the magnetosphere and that dissipated as atmospheric heating for the case of magnetospheric expansion, in a format similar to Fig. 8. Figure 14a shows co-latitude profiles of the power per unit area input to the magnetosphere assuming prompt response of the atmospheric flow, where the line and colour code follows Fig. 12. These powers remain relatively small and similar to each other for reasons already mentioned in Sect. 3.3. Integrated over the closed field region, the power is $101 \mathrm{TW}$ for the initial compressed steady state with a magnetopause at $45 R_{J}$ (red solid line), increasing to 266 and $237 \mathrm{TW}$, respectively, for the transient states expanded to 65 and $85 R_{J}$ (blue and black solid lines). The latter powers then reduce modestly to 179 and $189 \mathrm{TW}$ in the corresponding steady states (blue and black dashed lines). Values integrated over the closed field region per hemisphere are plotted versus $R_{M P}$ in Fig. 14b. The power input for the transient states (solid line) increases rapidly from the initial steady state for modest expansions of the system, before falling slowly with increasing magnetopause radius beyond $\sim 65 R_{J}$. These powers then fall by modest factors as the steady state is approached at a given radius (dashed line). The closed field magnetospheric power input in the transient states assuming an unresponsive atmosphere is shown by the dot-dashed line in Fig. 14b. This is roughly double that for the prompt atmospheric response as a result of the doubled Pedersen currents that then occur, shown in Fig. 13. The peak value is $\sim 480 \mathrm{TW}$ (per hemisphere) for an expansion to $\sim 65 R_{J}$. We recall from Sect. 3.3 that the essentially unchanging power integrated over each open field region is $\sim 23.5 \mathrm{TW}$.

The corresponding co-latitude profiles of power per unit area dissipated to atmospheric heating are shown in Fig. 14c, 

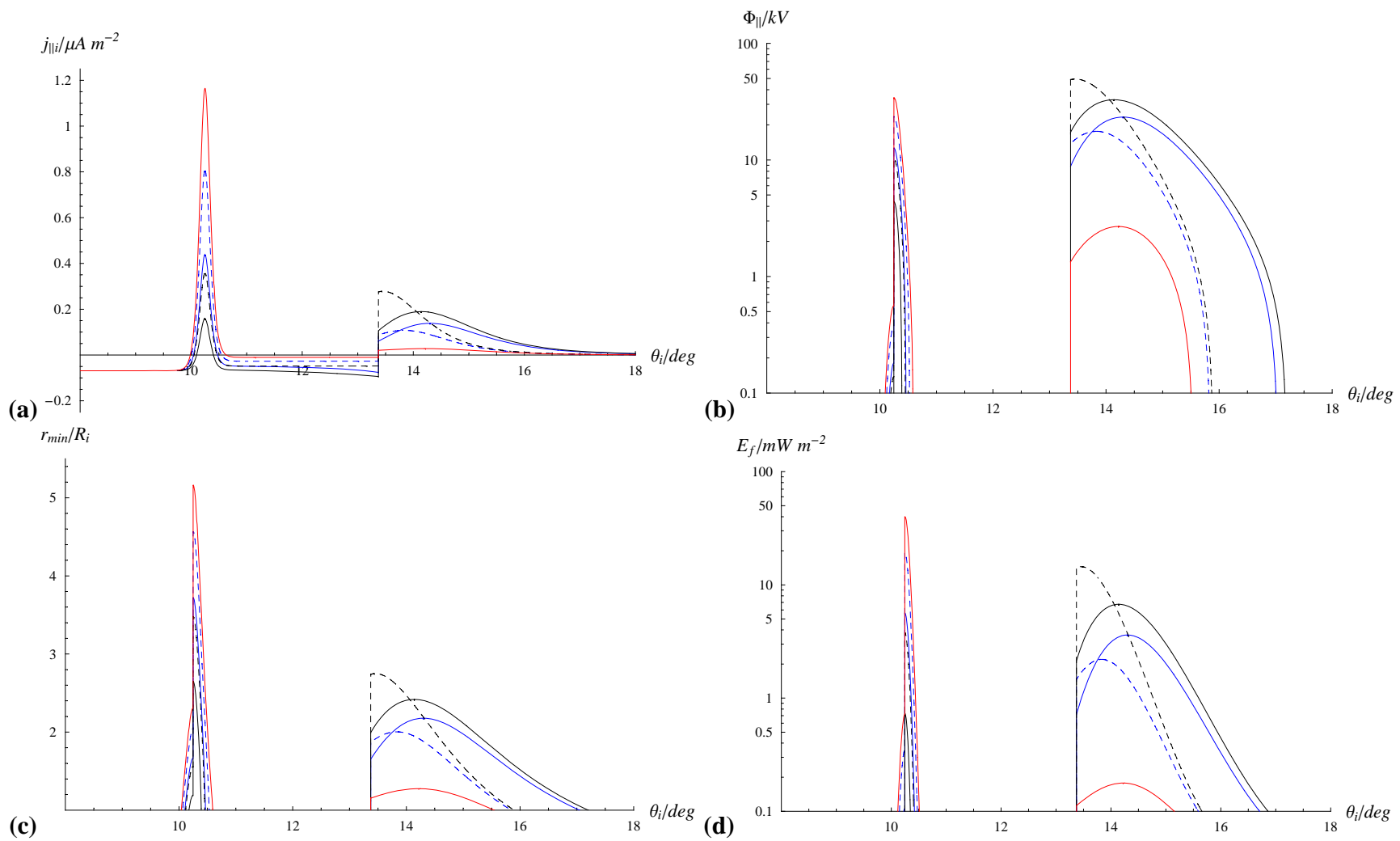

Fig. 15. Plots relating to auroral acceleration in regions of upward-directed field-aligned current for the case of magnetospheric expansion from an initial radius of $45 R_{J}$ to 65 and $85 R_{J}$, assuming a prompt atmospheric response to the changed plasma angular velocities. The line and colour codes follow Fig. 12. The parameters are plotted versus co-latitude over a reduced range from $8^{\circ}$ to $18^{\circ}$, spanning the open-closed field line boundary and the closed field region of interest. The panels show (a) the field-aligned current density as in Fig. 12d, (b) the accelerating voltage in regions of upward-directed field-aligned current employing a log scale, (c) the minimum radial distance of the acceleration region along the polar field lines, and (d) the precipitating electron energy flux again on a log scale.

again for the case of prompt atmospheric flow response. These can be seen to increase strongly with increasing subcorotation of the plasma. Integrated over the closed field region, the total powers are $14 \mathrm{TW}$ in the initial steady state, increasing to 256 and $427 \mathrm{TW}$ in the transient states as the system expands to 65 and $85 R_{J}$, before falling to 78 and $226 \mathrm{TW}$ in the corresponding steady states. Overall results in Fig. 14d, having the same format as Fig. 14b, show monotonic growth of the closed field power per hemisphere dissipated to atmospheric heating with the magnetospheric radius, in both the transient and steady states, reaching values of several hundred TW for large expansions in the case of an unresponsive atmospheric flow. We note that the essentially unchanging power integrated over each open field region is $\sim 195$ TW, as in Sect. 3.3.

\subsection{Auroral electron acceleration and precipitating energy flux}

\subsubsection{Auroral electron acceleration parameters for prompt atmospheric flow response case}

We now turn to the auroral precipitation associated with magnetospheric expansion, and in Fig. 15 show co-latitude profiles of relevant parameters for the case of prompt response of the atmospheric flow, similar to Fig. 9 for the case of compression. The line and colour format is the same as Fig. 12. Figure 15a reproduces the field-aligned currents shown previously in Fig. 14d, now shown over the reduced co-latitude range between $8^{\circ}$ and $18^{\circ}$, showing that for the case of system expansion, the upward currents remain separated into two zones, one corresponding to the open-closed field line boundary, the other to the middle magnetosphere. As the system expands the former currents are reduced, more so for the transient states than for the steady states, while the latter are enhanced. In all cases, however, acceleration of magnetospheric source electrons is required to carry the currents 

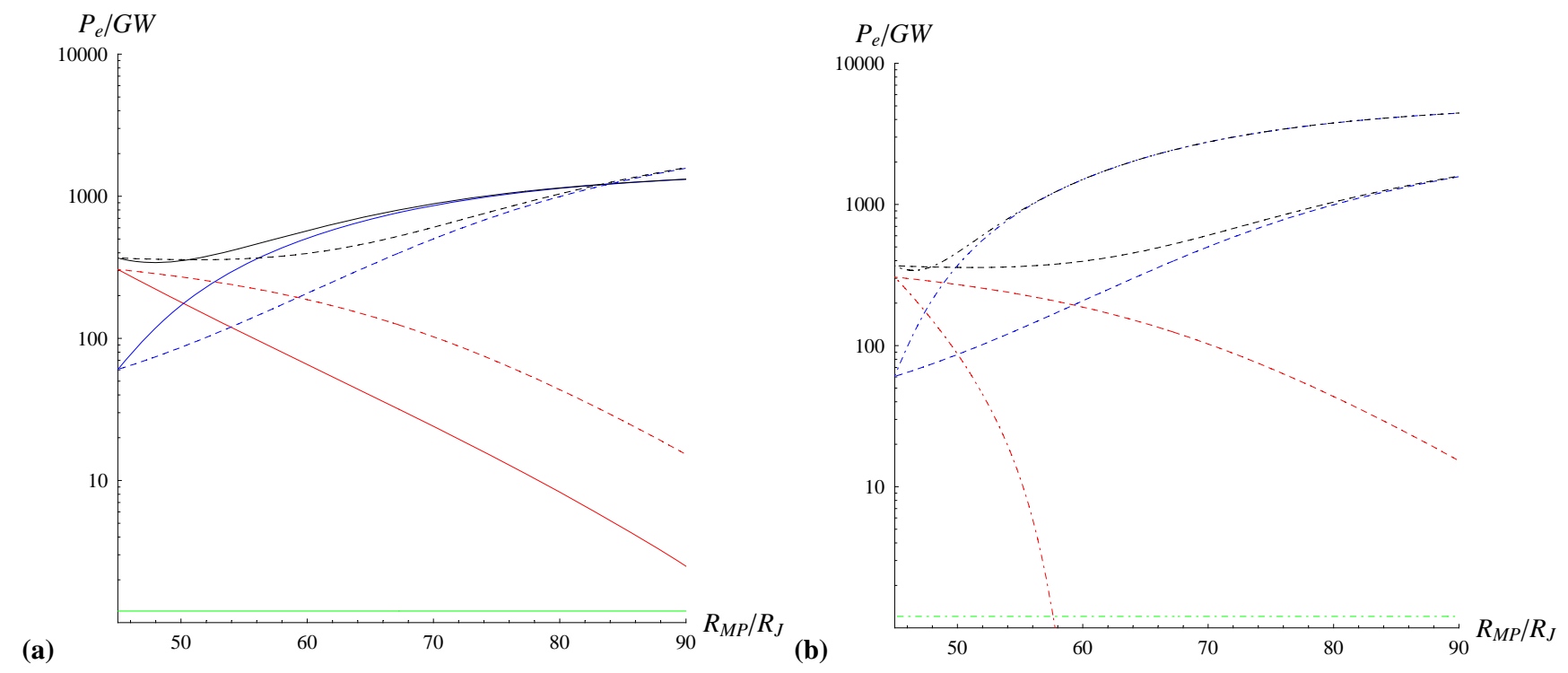

Fig. 16. (a) Overview of the variation of precipitating electron power per hemisphere (log scale) integrated over various regions of the model plotted versus the magnetopause radius $R_{M P}$ in $R_{J}$, for the case of system expansion from an initial magnetopause radius of $45 R_{J}$, assuming a prompt response of the atmospheric flow. Red lines show the power input into the open-closed field line boundary region (defined to be the region of total latitudinal width $0.75^{\circ}$ centred on the open-closed field line boundary), green lines the outer magnetosphere region, blue lines the middle magnetosphere region to $18^{\circ}$ co-latitude, while the black lines show the sum of these components. Solid lines refer to the transient states formed by rapid expansion outward from the initial magnetopause radius at $45 R_{J}$ (the left-hand border of the plot), while dashed lines show the steady-state values to which these revert after $\sim 1-2$ days. (b) As for panel (a) except for the case of an unresponsive atmospheric flow on the expansion time scale. In this case results for the transient states are plotted as dot-dashed lines. Results for the steady-states plotted for comparison as dashed lines are identical to panel (a).

according to the limiting values in Table 1 , though only just so for the magnetosheath source poleward of the open-closed boundary in the transient cases. It can be seen that as the current density falls at the open-closed field line boundary, so too do the field-aligned voltages (Fig. 15b), the minimum heights of the acceleration region (Fig. 15c), and the precipitating energy fluxes (Fig. 15d). Integrated over the whole boundary region (defined as in Sect. 3.4 above), the total precipitating electron powers fall from $306 \mathrm{GW}$ in the initial compressed steady state with a magnetopause at $45 R_{J}$, to 40 and $5 \mathrm{GW}$ for the transient expanded states with magnetopauses at 65 and $85 R_{J}$, respectively. The latter values then increase somewhat to 143 and $26 \mathrm{GW}$, respectively, in the corresponding steady states. The values for the middle magnetosphere then show the opposite behaviour, with the voltages, minimum acceleration region altitude, and energy fluxes all increasing with the expansion of the system. It is also notable that the acceleration region also widens to larger co-latitudes in the transient states compared with the steady states, though again this effect is exaggerated by the magnetic field model employed in the inner region, as indicated in Sect. 4.1. Integrated over the middle magnetosphere region to $18^{\circ}$, the total precipitating electron power is $60 \mathrm{GW}$ in the initial compressed steady state, increasing to $688 \mathrm{GW}$ and $1.24 \mathrm{TW}$ in the transient states with magnetopauses ex- panded to 65 and $85 R_{J}$, respectively. The latter values then change to $327 \mathrm{GW}$ and $1.28 \mathrm{TW}$ in the corresponding steady states.

\subsubsection{Overview of precipitating electron powers}

An overview of the variation of the precipitating electron power per hemisphere versus magnetopause radius for the case of system expansion from $45 R_{J}$ and prompt atmospheric response is shown in Fig. 16a, in the same format as Fig. 10a. The red, green, blue, and black lines thus refer to values integrated over the open-closed field line boundary region, the outer magnetosphere, the middle magnetosphere, and total values, respectively, with solid lines referring to the transient states and the dashed lines to the steady states. We thus note that the dashed lines corresponding to the steady states are identical to those shown previously in Fig. 10. As indicated by the examples discussed in Sect. 4.4.1 above, the precipitating electron power at the open-closed field line boundary (red lines) falls rapidly with expansion of the system in the transient states, before partially recovering in the steady states. The power input to the middle magnetosphere (blue lines) behaves oppositely over most of the range of magnetopause distances, increasing strongly with expansion of the system in the transient states, and then falling again in 
the steady states, though the transient values become comparable to and smaller than the steady-state values for the largest expansions, beyond $\sim 84 R_{J}$. We also note that the precipitating electron power into outer magnetosphere region (green line) remains at the unaccelerated value of $1.2 \mathrm{GW}$ throughout (given by Eq. 22), for both transient and steady states. The total precipitating electron power over all these regions combined (black lines) increases from $368 \mathrm{GW}$ in the compressed initial state with a magnetopause at $45 R_{J}$, to $1.24 \mathrm{TW}$ in the transient expanded state with magnetopause at $85 R_{J}$, the latter then increasing marginally to $1.31 \mathrm{GW}$ as steady conditions are resumed.

Corresponding results for the case of unresponding atmospheric flow on the expansion time scale are shown in Fig. 16b. The format follows Fig. 16a, except that the results for the transient cases are shown by the dot-dashed lines. It can be seen that the precipitating electron power into the open-closed field line boundary region in the transient case (dot-dashed red line) now falls to small values for modest expansions of the system, reaching an unaccelerated value of $0.45 \mathrm{GW}$ as the field-aligned current becomes downward-directed, for magnetopause radii exceeding $\sim 59 R_{J}$ (see Fig. 13d). On the other hand the electron power input to the middle magnetosphere in the transient cases increases very quickly with expansion of the system, reaching $\sim 4.15 \mathrm{TW}$ for an expansion to $85 R_{J}$. The transient input power in this case is $2-3$ times that for the transient states assuming prompt atmospheric response shown in Fig. 16a, and is typically $\sim 5$ times higher than the input power for the corresponding steady state.

\section{Discussion}

In this paper we have set up and investigated the consequences of a simple physically-motivated model of the response of the magnetospheric flow and resulting magnetosphere-ionosphere coupling current system at Jupiter to solar wind-induced compressions and expansions of the system. Although transitions between any two magnetospheric radii can be computed, to illustrate results we have considered compressions of an initially expanded system with a magnetopause radius of $85 R_{J}$, corresponding to a typical solar wind rarefaction region, inwards to radii down to $40 R_{J}$, corresponding to a strong solar wind compression region. We have also considered expansions of an initially compressed system with a magnetopause radius of $45 R_{J}$ outward to radii up to $90 R_{J}$. These are intended to represent the largest commonly-occurring solar wind-induced transitions that take place within the jovian system (e.g., Nichols et al., 2006). In this section we will summarise and discuss these results, beginning with the effects of solar windinduced compression.

The rather complicated behaviour of the system under compression is summarised for the auroral electron precipita- tion in Fig. 10, and for the power inputs to the magnetosphere and atmospheric heating in Figs. 8b and d, respectively, these figures being the principal reference points for the discussion given here. In an initially expanded steady state corresponding to a solar wind rarefaction region, the plasma angular velocity falls from near-rigid corotation in the inner magnetosphere to a significant degree of sub-corotation in the outer magnetosphere, before falling further (in the ionosphere) to small values on open field lines across the openclosed field line boundary. Upward-directed field-aligned currents thus flow at both the open-closed field line boundary and in the middle magnetosphere where the angular velocity falls with increasing latitude, both of which require acceleration of auroral electrons. A two-zone region of discrete auroral emission will therefore be produced, with emission intensities of order $\sim 100 \mathrm{kR}$, and total precipitating powers of $\sim 1 \mathrm{TW}$, dominated by the middle magnetosphere. With UV emission efficiencies of $\sim 10 \%$, the total UV output will thus be $\sim 100 \mathrm{GW}$, comparable to typical auroral UV power outputs inferred from both HST and Cassini data (Grodent et al., 2003a, b; Pryor et al., 2005). The power inputs to magnetospheric rotation in each hemisphere are $\sim 20 \mathrm{TW}$ on open field lines (essentially constant throughout) and $\sim 200$ TW on closed field lines, while that dissipated to atmospheric heating in each hemisphere is $\sim 200 \mathrm{TW}$ on open field lines (also essentially constant), and $\sim 100-200 \mathrm{TW}$ on closed field lines, increasing with the extension of the system and consequent sub-corotation of the plasma.

When the system is rapidly compressed inward from such a configuration, the plasma angular velocity initially rises towards rigid corotation on closed field lines for modest compressions, due to conservation of angular momentum. This increases the flow shear across the open-closed field line boundary, and hence the field-aligned current density and precipitating auroral electron power at the boundary, while decreasing that across the middle magnetosphere, where the field-aligned current and precipitating electron power are correspondingly reduced. Overall, the total precipitating electron power is reduced (Fig. 10). Similarly the power input to the magnetosphere and to atmospheric heating in the closed field line region are also reduced, ideally to zero in the case where the plasma exactly rigid corotates with the planet throughout, or corotates with the neutral atmosphere if the latter flow is unresponsive on this time scale. In the case illustrated in this paper, where the radius of the magnetopause in the initial steady state was taken to be $85 R_{J}$ (corresponding to a solar wind dynamic pressure of $\sim 0.02 \mathrm{nPa}$ ), this condition applies to compressions inward to $\sim 60 R_{J}$ $(\sim 0.09 \mathrm{nPa})$ in the case of prompt atmospheric response, or to $\sim 70 R_{J}(\sim 0.05 \mathrm{nPa})$ in the case of an unresponsive atmosphere. At this point the middle magnetosphere accelerated precipitation is switched off in the model, while weak accelerated precipitation in the outer magnetosphere begins (at intensities of a few $\mathrm{kR}$ ). The minimum total precipitating electron power is $\sim 250 \mathrm{GW}$ in this configuration (for both 
atmospheric response cases), then dominated by the emission from the open-closed field line boundary. The reduction in precipitating electron power from initial values of $\sim 1 \mathrm{TW}$ by typical factors of two or four in this case is the response to compression discussed originally by Southwood and Kivelson (2001) and Cowley and Bunce (2001). If the compression is halted within this radius (for a modest increase in solar wind dynamic pressure) then the precipitation at the open-closed field line boundary does not greatly change as sub-corotating conditions are resumed, while the usual accelerated precipitation in the middle magnetosphere region re-emerges, and once more becomes the dominant component. Overall, therefore, the precipitating electron power rises as steady-state conditions are resumed, while remaining less than that in the initial expanded steady state. Similar comments apply to both the power input to magnetospheric rotation, and into atmospheric heating.

As also noted by Southwood and Kivelson (2001) and Cowley and Bunce (2001), however, further rapid compression of the system to radii smaller than the above-mentioned limits raises the plasma angular velocity on closed field lines to conditions of super-corotation. In this case the flow shear, field-aligned current, and auroral precipitation at the open-closed field line boundary are further increased, while a fully-developed "reversed" current system is formed on closed field lines and grows in amplitude as the compression increases. The accelerated electron precipitation that then occurs in the outer magnetosphere peaks at the boundary with the middle magnetosphere (at typical intensities of several tens of $\mathrm{kR}$ in the model), thus being co-located with the poleward boundary of the middle magnetosphere precipitation in the steady-state. However, in this case the precipitation extends with diminishing intensity poleward of this boundary before brightening again at the open-closed field line boundary, thus in effect again forming a two-ring auroral system. As the compression increases, the precipitating electron power increases at both the open-closed field line boundary and in the outer magnetosphere region in the transient state, the former remaining modestly larger than the latter in the model (though again noting the intrinsic uncertainties in the former values associated with the width of the current layer at the open-closed field line boundary). The total precipitating electron power thus grows with increasing compression of the system under these conditions, reaching and exceeding the initial value of $\sim 1 \mathrm{TW}$ for compressions inside $\sim 50 R_{J}$ (corresponding to a solar wind dynamic pressure of $\sim 0.2 \mathrm{nPa}$ ) in the prompt atmospheric response case. For the unresponsive atmosphere case this condition is reached for a compression to $\sim 60 R_{J}(\sim 0.09 \mathrm{nPa})$, while for a compression e.g. to $\sim 45 R_{J}(\sim 0.3 \mathrm{nPa})$, the transient precipitating electron power is increased greatly to $\sim 5 \mathrm{TW}$. The super-corotating condition also greatly changes the related mechanical effects, since now the direction of power transfer is from the super-corotating magnetosphere to the planet. Part of this power is input to planet spin-up, while the re- mainder is dissipated as atmospheric heating, peaking at values of a few $\mathrm{W} \mathrm{m}^{-2}$ at the boundary between the outer and middle magnetosphere (Fig. 8c). The integrated heating rate on closed field lines can be raised to several hundred TW or more under these conditions. Such heating rates are expected to result in a significant transient thermospheric temperature response (see e.g., Melin et al., 2006). As sub-corotating steady-state conditions are resumed, the flow shear and precipitating electron power at the open-closed field line boundary falls, while the reversion of the closed field current system to the usual sense of flow results in the accelerated precipitation into the outer magnetosphere region being replaced by the usual accelerated middle magnetosphere precipitation, which, however, is of lesser intensity. Overall, therefore, the total precipitating electron power reduces to $\sim 400 \mathrm{GW}$ in the model as steady-state conditions are resumed under these compressed conditions, dominated by precipitation at the open-closed field line boundary. Similarly, power input to the sub-corotating closed field magnetosphere is resumed at reduced rates of $\sim 100 \mathrm{TW}$, while atmospheric heating rates on closed field lines are greatly reduced to a few tens of TW.

We finally summarise our results for rapid expansions of the system from such compressed modestly sub-corotating steady states, due to a sudden decrease in solar wind dynamic pressure. The discussion here is based largely on the overall results shown in Fig. 16 for the auroral electron precipitation, and in Figs. 14b and d for the power inputs to the magnetosphere and atmosphere. Expansion causes the plasma angular velocity to fall with increasing radial distance if angular momentum is conserved, to values less than that of the corresponding steady states. The shear in plasma velocity across the open-closed field line boundary is thus reduced compared with the initial state, and with it, the upward current and accelerated precipitation are also reduced at the boundary. The current can be reversed to downward and the accelerated precipitation extinguished for sufficient expansion in the case of an unresponsive atmosphere. At the same time, the flow shear across the middle magnetosphere is significantly increased on expansion, such that the upward-directed fieldaligned current is increased in this region, together with the accelerated electron precipitation. Overall, the total precipitating electron power is enhanced for significant expansions, to $\sim 1 \mathrm{TW}$ for an expansion to $\sim 70-90 R_{J}$ in the prompt atmosphere case, and to $\sim 3-4 \mathrm{TW}$ for similar expansions in the unresponsive atmosphere case, dominated by precipitation in the middle magnetosphere region. As steady-state conditions resume, precipitation into the middle magnetosphere region is reduced, while that into the open-closed field line boundary is enhanced, though remaining less than that in the middle magnetosphere for expansions beyond $\sim 60 R_{J}$ (falls in solar wind dynamic pressure below $\sim 0.09 \mathrm{nPa}$ ). Overall, the precipitating electron power is generally reduced in the steady state compared with the transient state, approaching values of $\sim 1 \mathrm{TW}$ as quoted at the beginning of this section. Similar behaviour also applies to the power input to 
the magnetosphere and to atmospheric heating. The power input to the magnetosphere on closed field lines rapidly increases as the plasma angular velocity drops in the transient states, from $\sim 100 \mathrm{TW}$ in the initial compressed steady state, to $\sim 250 \mathrm{TW}$ for expansions beyond $\sim 55 R_{J}$ in the case of prompt atmospheric response, $\sim 400 \mathrm{TW}$ in the case of an unresponsive atmosphere, before falling once more to stillelevated values as steady conditions are restored in the expanded state. The power input to the atmosphere on closed field lines also increases, from a few tens of TW in the initial compressed steady state, up to several hundred TW for significant expansions to $\sim 70-90 R_{J}$, before falling again to $\sim 100-200 \mathrm{TW}$ for significantly expanded conditions in the steady-state. Heating rates again peak at the boundary between the open and middle magnetosphere in the transient states, and may be expected to produce a significant thermospheric temperature response.

Overall, it can be seen that the model developed here anticipates a rich magnetospheric and atmospheric response of the jovian system to rapid compressions and expansions of the system induced by changes in the dynamic pressure of the solar wind. With regard to the issue of which process produces the short-lived intensifications of jovian UV emissions observed in Cassini data e.g. by Gurnett et al. (2002) and Pryor et al. (2005), our results indicate that in principle these could be due either to a strong rapid compression of the system from an expanded state which induces strong plasma super-corotation with respect to the neutral atmosphere (Fig. 10), or to a strong rapid expansion of the system from a compressed state which induces strong plasma sub-corotation (Fig. 16). In the former case the brightened emissions relate to the outer magnetosphere and open-closed field line boundary regions, while in the latter case they relate to the adjacent middle magnetosphere region at lower latitudes. Future systematic examination of remote sensing and in situ data will be of interest to determine the extent to which these expectations are realised.

Acknowledgements. Work at Leicester was supported by PPARC grant PPA/G/O/2003/00013 and PP/E000983/1. S. W. H. Cowley was supported by a Royal Society Leverhulme Trust Senior Research Fellowship, and J. D. Nichols by NASA grant HST-GO10862.01-A from the Space Telescope Science Institute to Boston University.

Topical Editor I. A. Daglis thanks two anonymous referees for their help in evaluating this paper.

\section{References}

Acuña, M. H., Behannon, K. W., and Connerney, J. E. P.: Jupiter's magnetic field and magnetosphere, in: Physics of the Jovian Magnetosphere, edited by: Dessler, A. J., Cambridge Univ. Press, Cambridge, UK, p. 1-50, 1983.

Bunce, E. J. and Cowley, S. W. H.: Divergence of the equatorial current in the dawn sector of Jupiter's magnetosphere: analysis of Pioneer and Voyager magnetic field data, Planet. Space Sci., 49, 1089-1113, 2001

Clarke, J. T., Ajello, J., Ballester, G., Jaffel, L. B., Connerney, J., Gérard, J.-C., Gladstone, G. R., Grodent, D., Pryor, W., Trauger, J., and Waite, J. H.: Ultraviolet auroral emissions from the magnetic footprints of Io, Ganymede, and Europa on Jupiter, Nature, 415, 997-1000, 2002

Clarke, J. T., Grodent, D., Cowley, S. W. H., Bunce, E. J., Zarka, P., Connerney, J. E. P., and Satoh, T.: Jupiter's auroras, in: Jupiter, edited by: Bagenal, F., Dowling, T. E., and McKinnon, W. B., Cambridge Univ. Press, Cambridge, UK, p. 639-670, 2004.

Connerney, J. E. P., Acuña, M. H., Ness, N. F., and Satoh, T.: New models of Jupiter's magnetic field constrained by the Io flux tube footprint, J. Geophys. Res., 103, 11 929-11 940, 1998.

Cowley, S. W. H. and Bunce, E. J.: Origin of the main auroral oval in Jupiter's coupled magnetosphere-ionosphere system, Planet. Space Sci., 49, 1067-1088, 2001.

Cowley, S. W. H. and Bunce, E. J.: Modulation of Jupiter's main auroral oval emissions by solar wind-induced expansions and compressions of the magnetosphere, Planet. Space Sci., 51, 57-79, 2003a.

Cowley, S. W. H. and Bunce, E. J.: Modulation of jovian middle magnetosphere currents and auroral precipitation by solar windinduced compressions and expansions of the magnetosphere: Initial conditions and steady state, Planet. Space Sci., 51, 31-56, 2003b.

Cowley, S. W. H., Nichols, J. D., and Bunce, E. J.: Distributions of current and auroral precipitation in Jupiter's middle magnetosphere computed from steady-state Hill-Pontius angular velocity profiles: Solutions for current sheet and dipole magnetic field models, Planet. Space Sci., 50, 717-734, 2002.

Cowley, S. W. H., Bunce, E. J., and Nichols, J. D.: Origins of Jupiter's main oval auroral emissions, J. Geophys. Res., 108(A4), 8002, doi:10.1029/2002JA009329, 2003a.

Cowley, S. W. H., Balogh, A., Dougherty, M. K., Dunlop, M. W., Edwards, T. M., Forsyth, R. J., Laxton, N. F., and Staines, K.: Plasma flow in the jovian magnetosphere and related magnetic effects: Ulysses observations, J. Geophys. Res., 101, $15197-$ $15210,1996$.

Cowley, S. W. H., Bunce, E. J., Stallard, T. S., and Miller, S.: Jupiter's polar ionospheric flows: theoretical interpretation, Geophys. Res. Lett., 30(5), 1220, doi:10.1029/2002GL016030, 2003b.

Cowley, S. W. H., Alexeev, I. I., Belenkaya, E. S., Bunce, E. J., Cottis, C. E., Kalegaev, V. V., Nichols, J. D., Prangé, R., and Wilson, F. J.: A simple axi-symmetric model of magnetosphereionosphere coupling currents in Jupiter's polar ionosphere, J. Geophys. Res., 110, A11209, doi:10.1029/2005JA011237, 2005.

Gong, B. and Hill, T. W.: Variations of jovian and saturnian auroras induced by changes in solar wind dynamic pressure, Magnetospheres of the Outer Planets 2005 Programme and Abstracts, p. 129, University of Leicester, Leicester, UK, 2005.

Gosling, J. T. and Pizzo, V. J.: Formation and evolution of corotating interaction regions and their three dimensional structure, Space Sci. Rev., 89, 21-52, 1999.

Grodent, D., Clarke, J. T., Kim, J., Waite Jr., J. H., and Cowley, S W. H.: Jupiter's main oval observed with HST-STIS, J. Geophys. Res., 108(A11), 1389, doi:10.1029/2003JA009921, 2003a. 
Grodent, D., Clarke, J. T., Waite Jr., J.H., Cowley, S. W. H., Gérard, J.-C., and Kim, J.: Jupiter's polar auroral emissions, J. Geophys. Res., 108(A10), 1366, doi:10.1029/2003JA010017, 2003b.

Gurnett, D. A., Kurth, W. S., Hospodarsky, G. B., Persoon, A. M., Zarka, P., Lecacheux, A., Bolton, S. J., Desch, M. D., Farrell, W. M., Kaiser, M. L., Ladreiter, H. P., Rucker, H. O., Galopeau, P., Louarn, P., Young, D. T., Pryor, W. R., and Dougherty, M. K.: Control of Jupiter's radio emission and aurorae by the solar wind, Nature, 415, 985-987, 2002.

Gustin, J., Gérard, J.-C., Grodent, D., Cowley, S. W. H., Clarke, J. T., and Grard, A.: Energy-flux relationship in the FUV jovian aurora deduced from HST-STIS spectral observations, J. Geophys. Res., 109, A10205, doi:10.1029/2003JA010365, 2004.

Hanlon, P. G., Dougherty, M. K., Krupp, N., Hansen, K. C., Crary, F. J., Young, D. T., and Toth, G.: Dual spacecraft observations of a compression event within the Jovian magnetosphere: Signatures of externally triggered super-corotation?, J. Geophys. Res., 109, A09S09, doi:10.1029/2003JA010116, 2004.

Hill, T. W.: Inertial limit on corotation, J. Geophys. Res., 84, 65546558, 1979.

Hill, T. W.: The jovian auroral oval, J. Geophys. Res., 106, 81018107, 2001.

Huang, T. S. and Hill, T. W.: Corotation lag of the jovian atmosphere, ionosphere and magnetosphere, J. Geophys. Res., 94, 3761-3765, 1989.

Huddleston, D. E., Russell, C. T., Kivelson, M. G., Khurana, K. K., and Bennett, L.: Location and shape of the jovian magnetopause and bow shock, J. Geophys. Res., 103, 20 075-20 082, 1998.

Isbell, J., Dessler, A. J., and Waite Jr., J. H.: Magnetospheric energization by interaction between planetary spin and the solar wind, J. Geophys. Res., 89, 10 716-10 722, 1984.

Kane, M., Mauk, B. H., Keath, E. P., and Krimigis, S. M.: Hot ions in Jupiter's magnetodisc: A model for Voyager-2 low-energy charged particle measurements, J. Geophys. Res., 100, 1947319486, 1995.

Khurana, K. K. and Kivelson, M. G.: Inference of the angular velocity of plasma in the jovian magnetosphere from the sweepback of magnetic field, J. Geophys. Res., 98, 67-79, 1993.

Kivelson, M. G. and Southwood, D. J.: Dynamical consequences of two modes of centrifugal instability in Jupiter's outer magnetosphere, J. Geophys. Res., 110, A12209, doi:10.1029/2003JA011176, 2005.

Knight, S.: Parallel electric fields, Planet. Space Sci., 21, 741-750, 1973.

Lundin, R. and Sandahl, I.: Some characteristics of the parallel electric field acceleration of electrons over discrete auroral arcs as observed from two rocket flights, in Symposium on European Rocket Research, ESA SP-135, ESA, Noordwijk, The Netherlands, p. 125, 1978.

Melin, H., Miller, S., Stallard, T., Smith, C., and Grodent, D.: Estimated energy balance in the jovian upper atmosphere during an auroral heating event, Icarus, 186, 256-265, 2006.

Millward, G., Miller, S., Stallard, T., Aylward, A. D., and Achilleos, N.: On the dynamics of the jovian ionosphere and thermosphere. III. The modelling of auroral conductivity, Icarus, 160, 95-107, 2002.

Millward, G., Miller, S., Stallard, T., Achilleos, N., and Aylward, A. D.: On the dynamics of the jovian ionosphere and thermosphere.
IV. Ion-neutral coupling, Icarus, 173, 200-211, 2005.

Nichols, J. D. and Cowley, S. W. H.: Magnetosphere-ionosphere coupling currents in Jupiter's middle magnetosphere: Dependence on the effective ionospheric Pedersen conductivity and iogenic plasma mass outflow rate, Ann. Geophys., 21, 1419-1441, 2003,

http://www.ann-geophys.net/21/1419/2003/.

Nichols, J. D. and Cowley, S. W. H.: Magnetosphere-ionosphere coupling currents in Jupiter's middle magnetosphere: Effect of precipitation-induced enhancement of the ionospheric Pedersen conductivity, Ann. Geophys., 22, 1799-1827, 2004, http://www.ann-geophys.net/22/1799/2004/.

Nichols, J. D. and Cowley, S. W. H.: Magnetosphere-ionosphere coupling currents in Jupiter's middle magnetosphere: Effect of magnetosphere-ionosphere decoupling by field-aligned auroral voltages, Ann. Geophys., 23, 799-808, 2005, http://www.ann-geophys.net/23/799/2005/.

Nichols, J. D., Cowley, S. W. H., and McComas, D. J.: Magnetopause reconnection rate estimates for Jupiter's magnetosphere based on interplanetary measurements at $~ 5 \mathrm{AU}$, Ann. Geophys., 24, 393-406, 2006,

http://www.ann-geophys.net/24/393/2006/.

Nichols, J. D., Bunce, E. J., Clarke, J. T., Cowley, S. W. H., Gérard, J. C., Grodent, D., and Pryor, W. R.: Response of Jupiter's UV auroras to interplanetary conditions as observed by the Hubble Space Telescope during the Cassini fly-by campaign, J. Geophys. Res., 112, A02203, doi:10.1029/2006JA012005, 2007.

Nishida, A. and Watanabe, Y.: Joule heating of the jovian ionosphere by corotation enforcement currents, J. Geophys. Res., 86, 9945-9952, 1981.

Pallier, L. and Prangé, R.: More about the structure of the high latitude jovian aurorae, Planet. Space Sci., 49, 1159-1173, 2001.

Phillips, J. L., Bame, S. J., Barraclough, B. L., McComas, D. J., Forsyth, R. J., Canu, P., and Kellog, P. J.: Ulysses plasma electron observations of the jovian magnetosphere, Planet. Space Sci., 41, 877-892, 1993a.

Phillips, J. L., Bame, S. J., Thomsen, M. F., Goldstein, B. E., and Smith, E. J.: Ulysses plasma electron observations in the jovian magnetosheath, J. Geophys. Res., 98, 21 189-21 202, 1993 b.

Pontius Jr., D. H.: Radial mass transport and rotational dynamics, J. Geophys. Res., 102, 7137-7150, 1997.

Pryor, W. R., Stewart, A. I. F., Esposito, L. W., McClintock, W. E., Colwell, J. E., Jouchoux, A. J., Steffl, A. J., Shemansky, D. E., Ajello, J. M., West, R. A., Hansen, C. J., Tsurutani, B. T., Kurth, W. S., Hospodarsky, G. B., Gurnett, D. A., Hansen, K. C., Waite, J. H., Crary, F. J., Young, D. T., Krupp, N., Clarke, J. T., Grodent, D., and Dougherty, M. K.: Cassini UVIS observations of Jupiter's auroral variability, Icarus, 178, 312-326, 2005.

Rego, D., Achilleos, N., Stallard, T., Miller, S., Prangé, R., Dougherty, M., and Joseph, R.: Supersonic winds in Jupiter's aurorae, Nature, 399, 121-124, 1999.

Rego, D., Prangé, R., and Gérard, J. C.: Lyman $\alpha$ and $\mathrm{H}_{2}$ bands from the giant planets: 1 . Excitation by proton precipitation in the jovian aurorae, J. Geophys. Res., 99, 17 075-17 094, 1994.

Scudder, J. D., Sittler Jr., E. C., and Bridge, H. S.: A survey of the plasma electron environment of Jupiter: a view from Voyager, J. Geophys. Res., 86, 8157-8179, 1981.

Smith, C. G. A., Miller, S., and Aylward, A. D.: Magnetospheric energy inputs into the upper atmospheres of the giant planets, 
Ann. Geophys., 23, 1943-1947, 2005,

http://www.ann-geophys.net/23/1943/2005/.

Southwood, D. J. and Kivelson, M. G.: A new perspective concerning the influence of the solar wind on Jupiter, J. Geophys. Res., 106, 6123-6130, 2001.

Stallard, T., Miller, S., Millward, G., and Joseph, R. D.: On the dynamics of the jovian ionosphere and thermosphere: 1 . The measurement of ion winds, Icarus, 154, 475-491, 2001.

Stallard, T. S., Miller, S., Cowley, S. W. H., and Bunce, E. J.: Jupiter's polar ionospheric flows: measured intensity and velocity variations poleward of the main auroral oval, Geophys. Res. Lett., 30(5), 1221, doi:10.1029/2002GL016031, 2003.
Vasyliunas, V. M.: Plasma distribution and flow, in: Physics of the Jovian Magnetosphere, edited by: Dessler, A. J., Cambridge Univ. Press, Cambridge, UK, p. 395-453, 1983.

Vasyliunas, V. M.: Role of the plasma acceleration time in the dynamics of the jovian magnetosphere, Geophys. Res. Lett., 21, 401-404, 1994.

Waite Jr., J. H., Cravens, T. E., Kozyra, J., Nagy, A. F., Atreya, S. K., and Chen, R. H.: Electron precipitation and related aeronomy of the jovian thermosphere and ionosphere, J. Geophys. Res., 88, 6143-6163, 1983. 